\title{
Evolution model for martensitic phase transformation in shape-memory alloys
}

\author{
TOMÁŠ ROUBÍČEK ${ }^{\dagger}$ \\ Mathematical Institute, Charles University, Sokolovská 83, \\ CZ-186 75 Praha 8, Czech Republic
}

[Received 6 October 2000 and in revised form 16 July 2001]

\begin{abstract}
A mesoscopical-level model for the evolution of microstructure in simple-laminate martensite undergoing an isothermal phase-transformation process within the context of a uniaxial deformation is proposed using a Hamiltonian approach to a relaxed problem involving a Young-measure-valued deformation gradient and Hill's maximum-dissipation principle involving positive homogeneous dissipation potential which reflects the energy needed for (and dissipated by) a phase transformation. A regularization by adding a (modified) volume-fraction gradient, which can be understood as a limit Ericksen-Timoshenko beam-like construction, is considered to ensure existence of a weak solution for a slow-process model. A numerical algorithm and computational experiments are also presented.
\end{abstract}

Keywords: Twinning; martensite; quasiplasticity; nonconvex scalar variational problems; double-well problem; relaxation; Young measures; evolution; dissipation; activation.

\section{Introduction}

Shape-memory alloys (SMAs) belong to so-called smart materials and have been the subject of intensive theoretical and experimental research over past decades. At low temperatures, SMAs crystallize typically in several low-symmetrical modes, monoclinic or tetragonal, called martensitic phases. This creates the phenomenon called twinning in the martensitic microstructure. In evolution, the martensitic phases transform to each other (and possibly also to a higher-symmetric phase- the so-called austenite). This martensitic transformation requires certain activation stress (typically tens of MPa) and thus a certain energy which is then dissipated. This process is rate independent and represents thus a certain plastic response of the material, beside the usual plasticity by slip which is, however, activated by much higher stress (typically hundreds of MPa). There is much physical literature confirming experimentally the above phenomena, e.g. Miyazaki [12] or Huo and Müller [16] and references therein.

An effective description of the microstructure in SMA can be given, depending on the purpose, at various levels. The classification of the levels is not understood in a unified manner in the literature but let us agree here to use the following convention. The macroscopical level deals only with an 'averaged' displacement $u$. The mesoscopical level deals with the macroscopical displacement $u$ together with an 'averaged' microstructure (counting, in particular, volume fraction of phases in question) described by a probability measure $v_{x}$ acting on the displacement gradient $\nabla u$ depending possibly on space (the variable $x$ ). The microscopical level deals with the displacement whose

Email: tomas.roubicek@mff.cuni.cz

Also at: Institute of Information Theory and Automation, Academy of Sciences, Pod vodárenskou věží 4 , CZ-182 08 Praha 8, Czech Republic. 


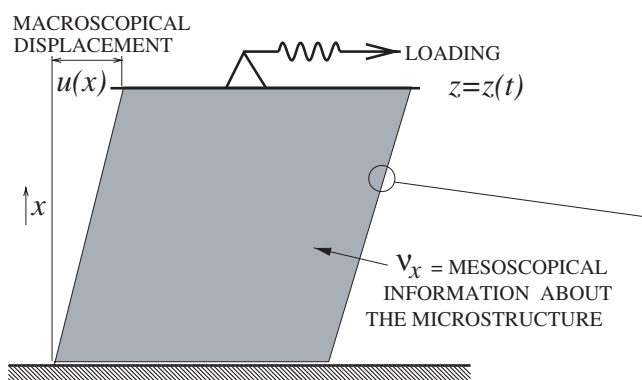

(a) Macro- or mesoscopical level

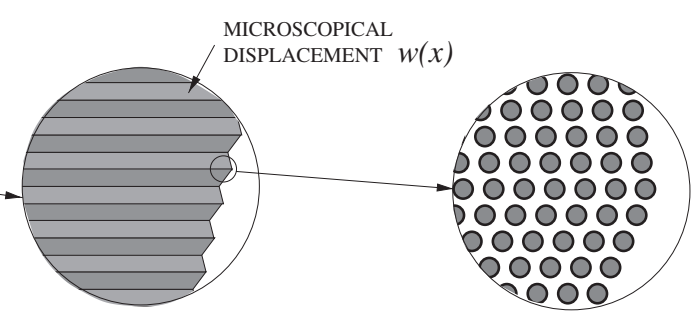

(b) Microscopical level (twined martensite) (c) Atomic level (schematic atomic grid)

FIG. 1. A one-dimensional uniaxial deformation of a single-crystal specimen of SMA (a) Macro- or mesoscopical level; (b) Microscopical level (twined martensite); (c) Atomatic level (schematic atomic grid).

gradient can oscillate fast at microscopical scales (typically about $10^{-5} \mathrm{~m}$ ) and we will denote it by $w$. As well as these continuum levels, on the finest scale we have the atomic level. Figure 1 illustrates this hierarchy.

In reality, higher-order laminated (or even more complicated) martensite often occurs but any rigorous approach to the evolution of such structures on the mesoscopical level seems difficult. This is why we deal with the simple-laminate situation as in Fig. 1(b), which will be sufficient for the effective and proper modelling of hysteretic phenomena. Moreover, our model concerns the isothermal case and single crystals often used in laboratry experiments, rather than the polycrystalic SMA used commercially. Further, we consider only scalar situations as in Fig. 1.

The aim of this paper is to present in detail a model on the mesoscopical level, and to prove the existence of its solution together with a convergence for numerical approximations. This model has been basically proposed (even in an anisothermal case but without rigorous analysis) in [34] modifying substantially the previous models [31,32,35] in the spirit of Srinivasa's remark [40] about the degree-one potential of dissipative forces routinely used in plasticity models. In Section 2, a detailed derivation is presented, together with a regularization by adding the gradient of volume fractions (viewed through phase indicators $\lambda_{\ell}$ introduced in Section 2.2), which can be understood as a limit Ericksen-Timoshenko beam-like construction. In Section 3, we justify this model for slow processes by a rigorous existence result for a suitably defined weak solution and specify the interesting connections with a steady-state model. The important result indicates that steady states need not minimize the elastic potential but are only Pareto optimal with respect to a certain class of potentials, in agreement with experiments but contrary to most mathematical literature (see e.g. $[2,3,5,23])$ addressing steady-state problems. In Section 4 , we describe an effective numerical algorithm and exhibit some computational experiments in a model case, showing desired hysteretic rate-independent response on cyclical external loading regimes.

Let us mention that a similar philosophy (but in a quite different setting) is found in the works of Mielke et al. (see [20,22] and some references therein), and also of Rajagopal and Srinivasa [26-28]. Other mesoscopical models have been developed by Frémond (see [12], investigated also by Colli et al. [7,8] and Hoffmann et al. [14]) and by James [17]; however, these models do not use a rate-independent law for evolution of the volume fraction of particular phases, which is not in agreement with experimental evidence $[12,16]$. Further, some sort of hysteretic response was shown to be possible in the context of mere nonlinear elasticity by Šilhavý [38] due to geometric incompatibility of particular phases. 

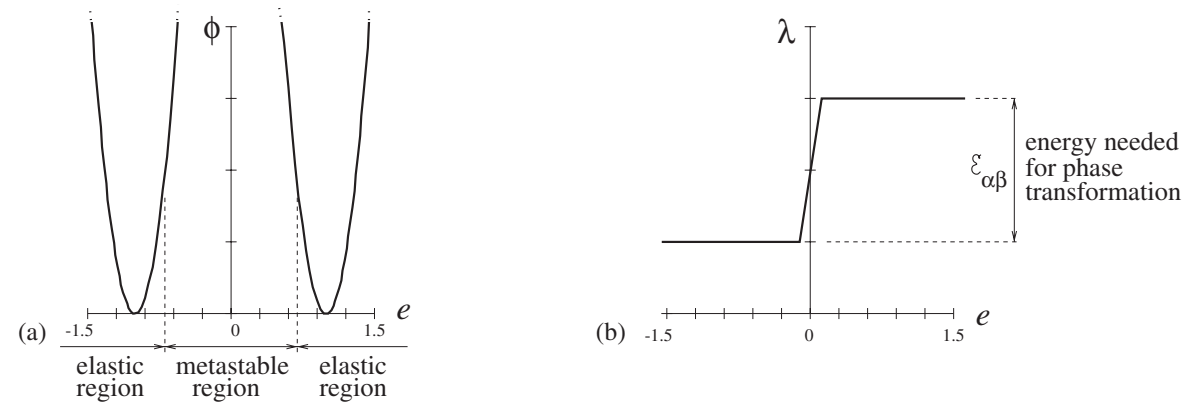

FIG. 2. (a) Double-well potential $\varphi(x, u, \cdot)$; (b) Phase indicator $\lambda$; see (4.3).

\section{A mesoscopical-level model}

In what follows, we consider as a body in its reference configuration a Lipschitz-bounded domain $\Omega \subset \mathbb{R}^{n}$. We confine ourselves to the case described by a scalar-valued displacement $u, w: \Omega \rightarrow \mathbb{R}$. This is sufficient to model the uniaxial shear deformation of interest to us, shown schematically in Figure 1 for the case $n=1$.

\subsection{Stored energy, Young-measure relaxation}

We first describe the structure of the stored energy. In terms of the microscopical displacement $w: \Omega \rightarrow \mathbb{R}$, the elastic stored energy (with possibly a body-force potential) of an inhomogeneous anisotropic material is considered as a local function of the displacement and the displacement gradient $\nabla w$ through a density $\varphi: \Omega \times \mathbb{R} \times \mathbb{R}^{n} \rightarrow \mathbb{R}$ :

$$
V_{0}(w):=\int_{\Omega} \varphi(x, w(x), \nabla w(x)) \mathrm{d} x .
$$

The situation depicted in Fig. 1(b) corresponds to the case when $\varphi(x, w,$.$) has two wells, each$ describing the elastic stored energy of one pure phase; then we can speak about a double-well potential (see Fig. 2(a)).

To be more specific, we will always assume that $\varphi$ is a Carathéodory function (i.e. $\varphi(x, \cdot, \cdot)$ continuous and $\varphi(\cdot, w, e)$ measurable) that satisfies the growth and coercivity of a polynomial degree $p>1$, i.e.

$$
\exists c_{1}, c_{0}>0 \quad \forall x \in \Omega, w \in \mathbb{R}, e \in \mathbb{R}^{n}: \quad c_{0}|e|^{p}-1 \leqslant \varphi(x, w, e) \leqslant c_{1}\left(1+|e|^{p}\right) .
$$

Then it is natural to consider $w$ ranging in $W^{1, p}(\Omega)$, the Sobolev space of functions whose distributional derivatives are $p$-integrable, i.e. $\nabla w \in L^{p}\left(\Omega ; \mathbb{R}^{n}\right)$. For such functions, $V_{0}$ is always finite and one can speak about traces on the boundary $\Gamma$ of $\Omega$, which makes it possible to incorporate the energy associated with the boundary and to define surface loading $f$ as functionals on $W^{1, p}(\Omega)$ defined by

$$
V(w):=V_{0}(w)+\int_{\Gamma} \psi(x, w(x)) \mathrm{d} S, \quad f(w):=\int_{\Gamma} g(x) w(x) \mathrm{d} S .
$$


If one were interested in steady-state solutions, one would seek displacements which minimize $V-$ $f$. This is meaningful in the context of elasticity especially if $\varphi(x, w, \cdot): \mathbb{R}^{n} \rightarrow \mathbb{R}$ is convex. If not, the solution need not exist (see [36] and references therein) and minimizing sequences then typically exhibit faster and faster oscillations in the strain. Many works consider a singular perturbation of $V_{0}$ by a higher-order term of capillarity-type, e.g.

$$
V_{\varepsilon}(w)=V_{0}(w)+\varepsilon \int_{\Omega}\left|\nabla^{2} w\right|^{2} \mathrm{~d} x
$$

which yields a minimizer $w_{\varepsilon}$ approximating (possibly in terms of subsequences) the macroscopical displacement, i.e.

$$
w_{\varepsilon} \rightarrow u \quad \text { weakly in } W^{1, p}(\Omega) \text { for } \varepsilon \rightarrow 0 .
$$

There are several ways in which we might try to avoid the microscopical level which is unsuitable for modelling more complex configurations. One is to construct a convex envelope $\varphi(x, u, e)^{* *}:=\inf _{w \in W_{0}^{1, p}(\Omega)} \int_{\Omega} \varphi(x, u, e+\nabla w) \mathrm{d} x$, where $W_{0}^{1, p}(\Omega)=\left\{w \in W^{1, p}(\Omega) ;\left.w\right|_{\Gamma}=\right.$ $0\}$, and consider the minimization of

$$
V^{\mathrm{c}}(u)-f(u)=\int_{\Omega} \varphi(x, u(x), \nabla u(x))^{* *} \mathrm{~d} x+\int_{\Gamma} \psi(x, u(x))-g(x) u(x) \mathrm{d} S .
$$

Under certain assumptions, one can interpret $u \in W^{1, p}(\Omega)$ minimizing (2.6) as a macroscopical displacement and relate it with the limit in (2.5). This coarse viewpoint (also called a coarse relaxation, see [33]) is, however, unsuitable for any attempt to determine the evolution of the microstructure because all the information about the fast oscillations is lost.

A finer relaxation can reflect and retain some essential information about the microstructure. The fast microscopical oscillations of $\nabla w_{\varepsilon}$ can be described ('in the limit' for $\varepsilon \rightarrow 0$ ) by a probability measure $v_{x}$ on $\mathbb{R}^{n}$ possibly depending (i.e. being parametrized) on $x \in \Omega$; see e.g. [33]. We then call $v=\left\{v_{x}\right\}_{x \in \Omega}$ a Young measure if, in addition, $x \mapsto v_{x}$ is weakly measurable. Very typically, the probability measure $v_{x}$ is composed only from a finite number of Dirac measures $\delta_{e}, e \in \mathbb{R}^{n}$, i.e.

$$
v_{x}=\sum_{\alpha=1}^{k} \gamma_{\alpha}(x) \delta_{e_{\alpha}(x)}, \quad \gamma_{\alpha}(x) \geqslant 0, \quad \sum_{\alpha=1}^{k} \gamma_{\alpha}(x)=1, \quad e_{\alpha}(x) \in \mathbb{R}^{n} .
$$

Usually, $k$ corresponds to the number of wells of $\varphi(x, u, \cdot)$ while each $e_{\alpha}(x)$ ranges over the neighbourhood of particular wells; in the general inhomogeneous case, $k=k(x)$. The information borne by such $v_{x}$ yields volume fractions $\gamma_{\alpha}$ of particular phases as well as strain $e_{\alpha}(x)$ of each particular phase at a given 'macroscopical' point $x \in \Omega$.

The assumption (2.2) allows one to prove rigorously that any minimizing sequence $\left\{w_{k}\right\}_{k \in \mathbb{N}}$ of $V-f$ does not concentrate energy in the sense that $\left\{\left|\nabla w_{k}\right|^{p} ; k \in \mathbb{N}\right\}$ as well as $\left\{\varphi\left(x, w_{k}, \nabla w_{k}\right) ; k \in \mathbb{N}\right\}$ are relatively weakly compact subsets of $L^{1}(\Omega)$; equally, $\left\{w_{\varepsilon}\right\}_{\varepsilon>0}$ from (2.5) shares this nonconcentration property, too. This suggests that a correct relaxation for this minimization problem can use the so-called $L^{p}$-Young measures $\mathcal{Y}^{p}\left(\Omega ; \mathbb{R}^{n}\right):=$ $\left\{v\right.$ is a Young measure; $\left.\int_{\Omega} \int_{\mathbb{R}^{n}}|e|^{p} v_{x}(\mathrm{~d} e) \mathrm{d} x<+\infty\right\}$ and, as in [33: chapter 5]. Without any misunderstanding, the extended stored energy will be denoted again by $V$. The relaxed problem 
then looks as

$$
\left.\begin{array}{ll}
\text { Minimize } & V(q)-\langle F, q\rangle \\
\text { subject to } & q \equiv(u, v) \in Q, \\
\text { where } & V(u, v):=\int_{\Omega} \int_{\mathbb{R}^{n}} \varphi(x, u(x), e) v_{x}(\mathrm{~d} e) \mathrm{d} x+\int_{\Gamma} \psi(x, u(x)) \mathrm{d} S, \\
& \langle F, q\rangle=f(u)=\int_{\Gamma} g(x) u(x) \mathrm{d} S, \text { and } \\
& Q:=\left\{(u, v) \in W^{1, p}(\Omega) \times \mathcal{Y}^{p}\left(\Omega ; \mathbb{R}^{n}\right): \nabla u(x)=\int_{\mathbb{R}^{n}} e v_{x}(\mathrm{~d} e), x \in \Omega\right\} .
\end{array}\right\}
$$

From now on, the couple $q \equiv(u, v) \in Q$ will be understood as a generalized configuration of the system.

To set up optimality conditions (see (2.14) below) and determine an evolution problem (see (2.21) or (3.1) below), we need to specify some linear structure on $Q$. Geometrically and topologically, the natural way is to embed $Q$ into a Banach space

$$
B:=W^{1, p}(\Omega) \times H^{*}
$$

via the embedding

$$
i: Q \rightarrow W^{1, p}(\Omega) \times H^{*}:(u, v) \mapsto\left(u, h \mapsto \int_{\Omega} h \bullet v \mathrm{~d} x\right)
$$

where $[h \bullet v](x):=\int_{\mathbb{R}^{n}} h(x, e) v_{x}(\mathrm{~d} e)$ for an integrand $h \in H$. Here, $H$ is a suitable linear space of integrands $h: \Omega \times \mathbb{R}^{n} \rightarrow \mathbb{R}$ (semi)normed by

$$
\|h\|:=\inf _{|h(x, e)| \leqslant a(x)+b|e|^{p}}\|a\|_{L^{1}(\Omega)}+b<+\infty .
$$

The adjective 'suitable' means that $H$ is large enough to contain all nonlinearities that can appear in the problem and simultaneously small enough to be separable under the seminorm (2.11). For example, consider

$$
\begin{aligned}
H:=\operatorname{span}\left\{h+\varphi \circ u+\lambda_{\ell}+\hat{e}_{i} ;\right. & h \in L^{1}\left(\Omega ; C_{0}\left(\mathbb{R}^{n}\right)\right), \\
& \left.u \in W^{1, p}(\Omega), \quad \ell=1, \ldots, L\right\},
\end{aligned}
$$

where $\varphi \circ u:(x, e) \mapsto \varphi(x, u(x), e)$ for any $u \in W^{1, p}(\Omega)$ and also $\hat{e}_{i}:(x, e) \mapsto e_{i}, i=1, \ldots, n$; a finite collection of nonlinearities $\lambda_{\ell}$ will be introduced in (2.16) below. In view of (2.2), $H$ contains at least one coercive integrand, which implies that the weak* closure of $i(Q)$, denoted by $\bar{Q}$, is a convex, metrizable, locally compact subset of $H^{*}$ (considered with its weak* topology) into which $W^{1, p}(\Omega)$ is embedded densely and homeomorphically via $u \mapsto i(u, d(\nabla u))$, with $[d(y)](x):=$ $\delta_{y(x)}$, see [33]. This makes $\bar{Q}$, called convex local compactification of $W^{1, p}(\Omega)$, a very natural envelope of $W^{1, p}(\Omega)$. Without confusion, we will identify $Q$ from (2.8) with its image $i(Q)$. Note that $V$ from (2.3) can be extended continuously not only to $Q$ but even to $\bar{Q}$, the extended functional being denoted, with no confusion, again by $V$.

With no great loss of applicability, we will assume

$$
\varphi(x, u, e)=\varphi_{0}(x, u)+\varphi_{1}(x, e), \quad \text { with } \varphi_{0}(x, \cdot) \text { and } \psi(x, \cdot) \text { from }(2.8) \text { convex. }
$$


Then, for $q=(u, \eta) \in B$, one has the simple formula $\bar{V}(q)=\left\langle\eta, \varphi_{1}\right\rangle+\int_{\Omega} \varphi_{0}(x, u) \mathrm{d} x+$ $\int_{\Gamma} \psi(x, u) \mathrm{d} S$ and one can see that the extended potential $V$ is convex even without imposing any convexity requirement on $\varphi_{1}$. Thus, even for nonconvex $\varphi_{1}$ like the double-well potential (4.1a), see also Fig. 2(a), the resulting relaxed problem is convex, i.e. the relaxed potential $V$ is convex and the newly arising the constraint $q \in Q$ is convex, too.

Having $Q$ embedded in the above manner, we can define in a standard way the normal cone $N_{Q}(q)$ to $Q$ at the point $q$. As $Q$ is dense in $\bar{Q}$, one has $N_{Q}(q)=N_{\bar{Q}}(q)$. The convexity of the relaxed problem allows us to write (2.8) equivalently in the form of the variational inclusion

$$
V^{\prime}(q)+N_{Q}(q) \ni F
$$

where $V^{\prime}(q)$ denotes the Gâteaux derivative of $V(q)$, given here by the formula

$$
\left[V^{\prime}(q)\right](\tilde{q})=\left\langle\tilde{\eta}, \varphi_{1}\right\rangle+\int_{\Omega} \frac{\partial \varphi_{0}}{\partial u}(x, u) \tilde{u} \mathrm{~d} x+\int_{\Gamma} \frac{\partial \psi}{\partial u}(x, u) \tilde{u} \mathrm{~d} S, \tilde{q}=(\tilde{u}, \tilde{\eta}),
$$

see [33: chapter 5] for details. Note that if we introduce $V_{Q}$ defined as $V(q)$ if $q \in Q$ and as $+\infty$ if $q \notin Q$, we can equally write (2.14) in the form $\partial V_{Q}(q) \ni F$ with ' $\partial$ ' denoting the subdifferential.

\subsection{Inelastic effects, rate of dissipation}

When the loading $f$ varies with time, the microstructure eventually may start to evolve. Experimentally it has repeatedly been proved that this evolution of microstructure is an activated process accompanied by dissipation that leads to a rate-independent hysteresis response of the material.

In fact, microstructural changes are activated by (a sufficiently large) temperature or stress. Thermally activated phase transformation is attributed to chaotic oscillations of atoms. Here we will consider only stress-activated transformations (under a constant temperature). One of the possible mechanisms may be associated with the evolution of dislocations: each real crystal contains a lot of dislocations in its atomic grid that can move quite easily thorough the body at very high speed (practically at the speed of sound) and, when running over a plane separating the two phases (see Fig. 1(c)), one atomic layer is switched from one phase to the other one so that the volume fraction of particular phases is slightly changed, i.e. $\gamma$ from (2.7) will then vary in time.

This activation phenomenon is intimately connected with rate-independent dissipation effects, with the nondifferentiability of the dissipative function as well as its positive degree-one homogeneity, and eventually with Hill's maximum-dissipation principle [13] known from classical plasticity, see (3.15) below. If we want to define the evolution $t \mapsto q(t) \equiv(u(t), v(t))$, we must also postulate the generalized impulse $\dot{q} \equiv(\dot{u}, \dot{v})$ with the dot indicating the time derivative, for which we need some geometric structure. This is, in fact, a matter of a certain choice and, as in [31], we take the convex geometry of $Q$ which will yield the desired response as shown in Section 4 below, (see also [34]). Then we take the dissipation function $R$ in the following form:

$$
R(\dot{q}):=\sum_{\ell=1}^{L} \int_{\Omega}\left|\lambda_{\ell} \bullet \dot{v}\right| \mathrm{d} x
$$

recall that we defined $[\lambda \bullet \dot{v}](x):=\int_{\mathbb{R}^{n}} \lambda(x, e) \dot{v}_{x}(\mathrm{~d} e)$. The functions $\lambda_{\ell}: \Omega \times \mathbb{R}^{n} \rightarrow \mathbb{R}$ reflect, on a mesoscopical level, a dissipation mechanism of the phase transformation; the simplest form of $\lambda_{\ell}$ is 
a function constant over each particular phase, i.e. in a neighbourhood of each well $e_{\alpha}$ of $\varphi(x, u, \cdot)$, and 'jumping continuously' in the metastable region. This suggests calling the $\lambda$ s phase indicators; sometimes, however, a notion of order parameters is introduced instead. Thus one can find that the energy $\mathcal{E}_{\alpha \beta}$ needed for phase transformation between the $\alpha$ th and $\beta$ th phases (per unit volume and at a given 'macroscopical point' $x$ ) equals

$$
\mathcal{E}_{\alpha \beta}(x)=\sum_{\ell=1}^{L}\left|\lambda_{\ell}\left(x, e_{\alpha}(x)\right)-\lambda_{\ell}\left(x, e_{\beta}(x)\right)\right|
$$

which allows us to set $\mathcal{E}_{\alpha \beta}$ with an arbitrary freedom; only one phase indicator $\lambda$ is needed for two phases, see Fig. 2(b), while three $\lambda$ s suffice for three phases, six $\lambda$ s for four phases, etc.

For the analysis below, we consider a certain regularization of the stored energy $V_{0}$ to control the spatial smoothness of $\lambda_{\ell} \bullet v, \ell=1, \ldots, L$. Inspired by the so-called Ericksen-Timoshenko beam, see Ren et al. [29,30] and referring to (2.4) for $V_{\varepsilon_{1}}$, we can consider

$$
\begin{array}{r}
V_{\varepsilon_{1}, \varepsilon_{2}}^{\rho}(w):=V_{\varepsilon_{1}}(w)+\int_{\Omega} \sum_{\ell=1}^{L}\left(\frac{1}{\varepsilon_{2}}\left(y_{\ell}-\lambda_{\ell}(\nabla w)\right)^{2}+\rho\left|\nabla y_{\ell}\right|^{2}\right) \mathrm{d} x \\
=\int_{\Omega} \varphi(x, w, \nabla w)+\varepsilon_{1}\left|\nabla^{2} w\right|^{2}+\sum_{\ell=1}^{L}\left(\frac{1}{\varepsilon_{2}}\left(y_{\ell}-\lambda_{\ell}(\nabla w)\right)^{2}+\rho\left|\nabla y_{\ell}\right|^{2}\right) \mathrm{d} x
\end{array}
$$

with $\rho>0$ a (small) regularization parameter preventing large spatial variations of volume fractions (measured in terms of the phase indicator $\lambda \mathrm{s}$ ) but still admit sharp interfaces in $\nabla w$. For the special case $L=d=1$ and $\lambda_{\ell}:(x, e) \mapsto e$, the stored energy (2.18) indeed coincides with EricksenTimoshenko beam, $\sqrt{\varepsilon_{2} \rho}$ being identified as an internal length scale in [29,30]. Thus, (2.18) can be viewed as the generalization of the Ericksen-Timoshenko model.

Letting $\varepsilon_{1} \rightarrow 0$, we arrive at the relaxed potential $V_{\varepsilon_{2}}^{\rho}(u, v):=V_{0}(u, v)+\int_{\Omega} \sum_{\ell=1}^{L}\left(\frac{1}{\varepsilon_{2}}\left(y_{\ell}-\right.\right.$ $\left.\left.\lambda_{\ell} \bullet v\right)^{2}+\rho\left|\nabla y_{\ell}\right|^{2}\right) \mathrm{d} x$ and, letting furthermore $\varepsilon_{2} \rightarrow 0$ push the differences $\left\{y_{\ell}-\lambda_{\ell} \bullet \nu\right\}_{\ell=1}^{L}$ penalized in the $L^{2}\left(\Omega ; \mathbb{R}^{L}\right)$-norm to zero, we eventually get

$$
V^{\rho}(u, v):=V_{0}(u, v)+\rho \sum_{\ell=1}^{L}\left\|\nabla\left(\lambda_{\ell} \bullet v\right)\right\|_{L^{2}\left(\Omega ; \mathbb{R}^{n}\right)}^{2}+\int_{\Gamma} \psi(x, u) \mathrm{d} S .
$$

From now on, we work with this regularized relaxed stored energy $V^{\rho}$ rather than $V$, denoting again by $V_{Q}^{\rho}(q)$ the potential $V^{\rho}(q)$ augmented by the indicator function of $Q$, i.e. $V_{Q}^{\rho}(q)=V^{\rho}(q)$ if $q \in Q$ and $V_{Q}^{\rho}(q)=+\infty$ if $q \notin Q$. Again, $V_{Q}^{\rho}: W^{1, p}(\Omega) \times H^{*} \rightarrow \mathbb{R} \cup\{+\infty\}$ is convex.

\subsection{Inertial effects, kinetic energy}

It is natural to define the kinetic energy as the quadratic form

$$
T_{\text {kin }}(\dot{q}):=\frac{1}{2} \int_{\Omega} \varrho(x)|\dot{u}|^{2} \mathrm{~d} x
$$

where $\varrho(x)>0$ is the mass density. Note that only the velocity and not the time rate of the microstructure $\dot{v}$ contributes to the kinetic energy because only displacement actually transports the mass. In other words, no microstructural inertia is considered. 
Of course, the presence of inertial effects makes the response always rate-dependent. Nevertheless, except in extremely fast processes like that considered by Lapczyk et al. [19], these effects may as well be neglected, and this we shall do in Sections 3 and 4.

\subsection{The dynamics, Hamiltonian framework}

The evolution $t \mapsto q(t)$ is governed by the following evolution inclusion:

$$
T_{\text {kin }}^{\prime} \frac{\mathrm{d}^{2} q}{\mathrm{~d} t^{2}}+\partial R\left(\frac{\mathrm{d} q}{\mathrm{~d} t}\right)+\left[V^{\rho}\right]^{\prime}(q)+N_{Q}(q) \ni F(t), \quad q(0)=q_{0}, \quad \dot{q}(0)=p_{0},
$$

where $T_{\text {kin }}^{\prime}$ is the linear operator being the differential of the quadratic form $T_{\text {kin }}$, while $\partial R$ denotes the subdifferential of $R$ which is a set-valued monotone mapping. Also, $q_{0} \equiv\left(u_{0}, v_{0}\right)$ is the initial configuration while $p_{0}$ is the initial impulse whose only first component is relevant because (2.20) depend only on $\dot{u}$ but not $\dot{v}$. This inclusion can be derived from the Hamilton variational principle adapted for nonconservative systems, see also Bedford [4], which says that the integral

$$
\int_{0}^{T} T_{\mathrm{kin}}(\dot{q})-V_{Q}^{\rho}(q)+\left\langle F+F_{\mathrm{N}}, q\right\rangle \mathrm{d} t
$$

is stationary with $V_{Q}^{\rho}$ defined at the end of Section $2.2, F$ defined in (2.8), and with $F_{\mathrm{N}}=R^{\prime}(\dot{q})$ a nonconservative force considered, just for derivation of stationarity of (2.22), as fixed. In our case, $R$ is nondifferentiable, so that the last equality turns out rather to the inclusion $F_{\mathrm{N}} \in \partial R(\dot{q})$.

For $R$ quadratic (i.e. $\partial R$ single-valued and even linear), this model has been proposed in [31]; we refer also to [15] for non-isothermal processes.

\subsection{Relation with static models}

Our model (2.21) is, to some extent, consistent with the standard 'mesoscopical' steady-state model (2.8) in the sense that, if the loading $f=f(t)$ is constant and if

$$
\partial R(0)=\{0\},
$$

any stationary point $q$ of (2.21) must obviously solve (2.14) which is, on the condition (2.13), just equivalent to the minimization of the energy (2.8). Let us remark that (2.23) means, in particular, that $R$ is Gâteaux differentiable at 0 . In this situation, one can immediately ask a question whether any trajectory $q=q(t)$ minimizes the energy. The mathematically rigorous answer is not clear for the full model (2.21), though it holds for the quasistationary case $T_{\text {kin }}=0$, see Section 3.3. This consistency represents a certain justification of (2.21) and simultaneously makes the range of validity the steady-state model (2.8) more explicit in terms of the conditions (2.13) and (2.23). Yet, let us emphasize that (2.23) just excludes non-trivial homogeneous degree-one potentials $R$ which are necessary to model rate-independent dissipation mechanisms which are, in turn, experimentally observed.

\section{A rate-independent model for slow processes}

The mathematical analysis of the second-order inclusion (2.21) is quite complicated and also the numerical response, which is necessarily rate dependent, would not be very illustrative. For these 
reasons, we focus on slow processes and both neglect the inertial effects by putting $T_{\text {kin }}=0$ and consider the dissipation potential $R$ positively degree-one homogeneous, e.g. $R$ from (2.16). In fact, $T_{\text {kin }}$ plays a role only within extremely fast processes [19], as already mentioned.

Thus, we consider instead of (2.21) only the following first-order inclusion:

$$
\partial R\left(\frac{\mathrm{d} q}{\mathrm{~d} t}\right)+\left[V^{\rho}\right]^{\prime}(q)+N_{Q}(q) \ni F(t), \quad q(0)=q_{0} .
$$

More specifically, in view of the special case (2.16) we write $R$ in the form

$$
\begin{aligned}
& R=\Psi \circ P^{*} \quad \text { with } \quad \Psi: L^{1}\left(\Omega ; \mathbb{R}^{L}\right) \rightarrow \mathbb{R}, \\
& P: L^{p_{1}}\left(\Omega ; \mathbb{R}_{p_{2}}^{L}\right) \rightarrow W^{1, p}(\Omega)^{*} \times H .
\end{aligned}
$$

Of course, $P^{*}: B \rightarrow L^{p_{1} /\left(p_{1}-1\right)}\left(\Omega ; \mathbb{R}_{p_{2} /\left(p_{2}-1\right)}^{L}\right)$ denotes the adjoint operator to $P$; we denote by $\mathbb{R}_{p}^{L}$ the Euclidean space endowed with the norm $\left|\left(a_{1}, \ldots, a_{L}\right)\right|_{p}=\left(\sum_{\ell=1}^{L}\left|a_{\ell}\right|^{p}\right)^{1 / p}$ for $1 \leqslant p<$ $+\infty$, or $\left|\left(a_{1}, \ldots, a_{L}\right)\right|_{\infty}=\max _{\ell=1}^{L}\left|a_{\ell}\right|$. For example, $|\cdot|_{2}$ is the usual Euclidean norm. Let us agree to specify the norm on $\mathbb{R}^{L}$ only whenever essential. The form (3.2) indeed covers (2.16) if one takes the linear continuous operator $P: L^{1}\left(\Omega ; \mathbb{R}^{L}\right) \rightarrow W^{1, p}(\Omega)^{*} \times H$ and the functional $\Psi: L^{1}\left(\Omega ; \mathbb{R}^{L}\right) \rightarrow \mathbb{R}$ as

$$
P g=\left(0, \sum_{\ell=1}^{L} g_{\ell} \cdot \lambda_{\ell}\right) \quad \text { and } \quad \Psi=\|\cdot\|_{L^{1}\left(\Omega ; \mathbb{R}_{1}^{L}\right)} .
$$

Note that we took $p_{1}=p_{2}=1$ because the phase indicators $\lambda_{\ell}$ are assumed bounded so that $g_{\ell} \cdot \lambda_{\ell}$, defined by $(x, s) \mapsto g_{\ell}(x) \lambda_{\ell}(x, s)$, has a finite norm (2.11) for any $g_{\ell} \in L^{1}(\Omega)$. Then $P^{*} q$ belongs even to $L^{\infty}\left(\Omega ; \mathbb{R}^{L}\right)$. In fact, it holds that

$$
P^{*}(u, v)=\left(\lambda_{\ell} \bullet v\right)_{\ell=1, \ldots, L}
$$

so that (3.3) actually gives (2.16). Moreover, for $\omega \in L^{\infty}\left(\Omega ; \mathbb{R}^{L}\right)$ we always have $P^{* *} \omega=P \omega \in$ $B^{\prime}$, which allows us to work with $P$ instead of $P^{* *}$ and also with $B^{\prime}$ instead of a slightly mysterious dual $B^{*}$.

Doubly nonlinear problems of the type (3.1) have been investigated, for example, by Colli and Visintin [9] (see also [41: Section III.2]) from which Sections 3.1 and 3.2 are inspired. The peculiarity of the problem (3.1)-(3.3) is that, in contrast to [9,41], degeneration (i.e. $P$ has no inverse) and concentration (caused by the $L^{1}$-structure of $\Psi$ ) may occur. These phenomena are, however, essential to model the rate-independent response of SMA. Moreover, it does not seem possible to perform analysis in the conventional measure-theoretical framework (see also Mielke and Theil [21] for an alternative approach to a similar, doubly nonlinear problem), which forces us to use the many technicalities related with finitely additive measures below.

\subsection{Weak formulation}

Let us denote by $L_{\mathrm{w}}^{\infty}(0, T ; B)$ the Banach space of essentially bounded and weakly* measurable functions $q:(0, T) \rightarrow B$, i.e. $t \mapsto\langle q(t), v\rangle$ is Lebesgue measurable for any $v \in B^{\prime}$, with $B^{\prime}=W^{1, p}(\Omega)^{*} \times H$ denoting the predual of $B$, i.e. $B=\left(B^{\prime}\right)^{*}$. Let us recall that 
$L_{\mathrm{w}}^{\infty}(0, T ; B) \neq L^{\infty}(0, T ; B)$ in general, see Fattorini [11: Example 12.2.8]; the equality would hold if $B$ were separable, see [11: Examples 5.0.39 and 12.9.6]. If $B$ has a separable predual $B^{\prime}=W^{1, p}(\Omega)^{*} \times H$ (as assumed), the norm $\|q\|_{L_{\mathrm{w}}^{\infty}(0, T ; B)}$ can be defined as ess $\sup _{t \in[0, T]}\|q(t)\|_{B}$, see [11: Lemma 12.2.2]. Also, $L_{\mathrm{w}}^{\infty}(0, T ; B) \cong L^{1}\left(0, T ; B^{\prime}\right)^{*}$, see [11: Theorem 12.2.11].

Let us still abbreviate $\Omega_{T}:=\Omega \times(0, T)$ and $\bar{\Omega}_{T}$ the closure of $\Omega_{T}$. Moreover, we will use the Banach space $\operatorname{vba}\left(\Omega_{T} ; \mathbb{R}^{L}\right) \cong L^{\infty}\left(\Omega_{T} ; \mathbb{R}^{L}\right)^{*}$, the abbreviation 'vba' indicating 'bounded additive set functions vanishing on Lebesgue-zero-measure sets', i.e. certain finitely additive measures, see Yosida and Hewitt [42]. Instead of $\int_{\Omega_{T}} v(x, t) \mu(\mathrm{d} x \mathrm{~d} t)$ we write simply $\langle\mu, v\rangle_{\Omega_{T}}$. The regularization (2.19) can be written in terms of $P$ as follows:

$$
V_{Q}^{\rho}(q)=V_{Q}(q)+\rho\left\|\nabla P^{*} q\right\|_{L^{2}\left(\Omega ; \mathbb{R}^{n \times L}\right)}^{2} .
$$

Moreover, we denote

$$
\mathcal{G}(t) \equiv\left[\mathcal{G}\left(V^{\rho}, Q, F, q\right)\right](t):=V_{Q}^{\rho}(q(t))-\langle F(t), q(t)\rangle
$$

the total Gibbs energy at a current time $t$. We will furthermore assume, as well as (2.2) with (2.13), the following data qualification:

$$
\begin{gathered}
\psi(x, u) \geqslant c_{2}|u|^{2}, \\
F \in W^{1,1}\left(0, T ; B^{*}\right), \\
q_{0} \in Q, \quad V_{Q}^{\rho}\left(q_{0}\right)-\left\langle F(0), q_{0}\right\rangle=\min _{q \in Q}\left(V_{Q}^{\rho}(q)-\langle F(0), q\rangle\right) .
\end{gathered}
$$

The degenerate doubly nonlinear inclusion (3.1) represents two mutually coupled variational inequalities, which is reflected in the following definition.

Definition $1 q \in L_{\mathrm{w}}^{\infty}(0, T ; B)$ will be a weak solution of the problem (3.1)-(3.2) if

$$
\begin{aligned}
& \exists \omega \in L^{\infty}\left(\Omega_{T} ; \mathbb{R}^{L}\right) \quad \exists z \in L_{\mathrm{w}}^{\infty}\left(0, T ; B^{*}\right) \quad \exists \mu \in \operatorname{vba}\left(\Omega_{T} ; \mathbb{R}^{L}\right): \\
& q(0)=q_{0}, \quad P^{*} q \in L^{2}\left(0, T ; H^{1}\left(\Omega ; \mathbb{R}^{L}\right)\right), \\
& \exists \lim _{t \searrow 0} \mathcal{G}(t)=\mathcal{G}(0), \quad \exists \lim _{t \nearrow T} \mathcal{G}(t)=\mathcal{G}(T), \\
& P \omega+z=F,\left.\quad \mu\right|_{C\left(\bar{\Omega}_{T} ; \mathbb{R}^{L}\right)}=P^{*} \frac{\mathrm{d} q}{\mathrm{~d} t} \text { in the sense of distributions, } \\
& \int_{0}^{T}\langle\xi, v\rangle-\langle\omega, v\rangle-\left\langle\frac{\mathrm{d} F}{\mathrm{~d} t}, q\right\rangle \mathrm{d} t-\langle\mu, \xi\rangle_{\Omega_{T}} \geqslant \mathcal{G}(T)-\mathcal{G}(0) \\
& \quad \forall v \in L^{1}\left(\Omega_{T} ; \mathbb{R}^{L}\right), \xi \in L^{\infty}\left(\Omega_{T} ; \mathbb{R}^{L}\right), \xi \in \partial \Psi(v), \\
& \int_{0}^{T}\langle z-\xi, q-v\rangle \mathrm{d} t \geqslant 0 \quad \forall v \in L^{1}(0, T ; B), \xi \in L_{\mathrm{w}}^{\infty}\left(0, T ; B^{*}\right), \xi \in \partial V_{Q}^{\rho}(v) .
\end{aligned}
$$

The following assertion justifies the above definition at least in the case when the Gibbs energy variation $\frac{\mathrm{d}}{\mathrm{d} t} \mathcal{G}$, the external-force work $\left\langle F(\cdot), \frac{\mathrm{d}}{\mathrm{d} t} q\right\rangle$, as well as the dissipation rate $\mu$ do not concentrate.

Proposition 1 (Relation with (3.1)-(3.2).) The inequality (3.8f) simply means

$$
z \in \partial V_{Q}^{\rho}(q) .
$$


Moreover, if one assumes that the following regularity (i.e. nonconcentration) hypotheses

$$
\mathcal{G}, \quad\left\langle F(\cdot), \frac{\mathrm{d} q}{\mathrm{~d} t}\right\rangle, \quad \text { and } \mu \text { are absolutely continuous }
$$

as functions on $[0, T]$ and as a measure on $\Omega_{T}$, respectively, then the inequality (3.8e) means $\omega \in$ $\partial \Psi\left(P^{*} \frac{\mathrm{d}}{\mathrm{d} t} q\right)$ and $q$ satisfies the inclusion (3.1)-(3.2).

Proof. First, (3.8f) simply means (3.9) due to maximal monotonicity of $\partial V_{Q}^{\rho}$. Furthermore, we prove that (3.8e) means $\omega \in \partial \Psi\left(P^{*} \frac{\mathrm{d} q}{\mathrm{~d} t}\right)$ at least if (3.10) holds because then, using also (3.7c), one can write

$$
\begin{aligned}
\mathcal{G}(0)-\mathcal{G}(T)- & \int_{0}^{T}\left\langle\frac{\mathrm{d} F}{\mathrm{~d} t}, q\right\rangle \mathrm{d} t=\int_{0}^{T}\left\langle F-z, \frac{\mathrm{d} q}{\mathrm{~d} t}\right\rangle \mathrm{d} t \\
& =\int_{0}^{T}\left\langle P \omega, \frac{\mathrm{d} q}{\mathrm{~d} t}\right\rangle \mathrm{d} t=\int_{0}^{T}\left\langle\omega, P^{*} \frac{\mathrm{d} q}{\mathrm{~d} t}\right\rangle \mathrm{d} t=\langle\mu, \omega\rangle_{\Omega_{T}}
\end{aligned}
$$

provided we have chosen $z=F-P \omega$ as we can because of (3.9). We also used that, for this $z$, it holds that $\frac{\mathrm{d}}{\mathrm{d} t} V_{Q}^{\rho}(q(t))=\left\langle z, \frac{\mathrm{d}}{\mathrm{d} t} q\right\rangle$ because, due to convexity of $V_{Q}^{\rho}$, one has

$$
\begin{aligned}
\frac{V_{Q}^{\rho}(q(t+\varepsilon))-V_{Q}^{\rho}(q(t))}{\varepsilon} & \geqslant\left\langle z(t), \frac{q(t+\varepsilon)-q(t)}{\varepsilon}\right\rangle \\
& =\left\langle F(t), \frac{q(t+\varepsilon)-q(t)}{\varepsilon}\right\rangle-\left\langle\omega(t), P^{*} \frac{q(t+\varepsilon)-q(t)}{\varepsilon}\right\rangle
\end{aligned}
$$

and similarly

$$
\frac{V_{Q}^{\rho}(q(t))-V_{Q}^{\rho}(q(t-\varepsilon))}{\varepsilon} \leqslant\left\langle F(t), \frac{q(t)-q(t-\varepsilon)}{\varepsilon}\right\rangle-\left\langle\omega(t), P^{*} \frac{q(t)-q(t-\varepsilon)}{\varepsilon}\right\rangle
$$

for any $\varepsilon>0$ so that the desired equality $\frac{\mathrm{d}}{\mathrm{d} t} V_{Q}^{\rho}(q(t))=\left\langle z, \frac{\mathrm{d}}{\mathrm{d} t} q\right\rangle$ can be obtained by passing $\varepsilon \searrow 0$, see also [6: Lemma 3.3]; note that, for a.a. $t \in[0, T]$, the limits exist due to the assumption (3.10) together with $(3.8 d)$ which also ensures that $P^{*} \frac{\mathrm{d}}{\mathrm{d} t} q$ is absolutely continuous. Thus, by (3.11) one can see that $(3.8 e)$ results in

$$
\langle\xi-\omega, v-\mu\rangle_{\Omega_{T}} \geqslant 0
$$

which just gives $\omega \in \partial \Psi(\mu)$. This means $\omega \in \partial \Psi\left(P^{*} \frac{\mathrm{d}}{\mathrm{d} t} q\right)$, from which one can get $\omega \otimes \lambda \in$ $\partial \tilde{R}\left(\frac{\mathrm{d}}{\mathrm{d} t} \nu\right)$ by elementary calculus. Thus $P \omega+z=F$ together with (3.9) and with $\partial R=P \circ \partial \Psi \circ P^{*}$ (which is ensured by continuity of $\Psi$ at some, here even each, point of $\operatorname{Range}\left(P^{*}\right)$ ) eventually gives $\partial R\left(\frac{\mathrm{d}}{\mathrm{d} t} q\right)+\partial V_{Q}^{\rho}(q) \ni F$.

In view of (3.4) and (3.8d), $\left|\mu_{\ell}(t, x)\right|$ represents the rate of the lth-transformation (at a current point $\left.(t, x) \in \Omega_{T}\right)$ while $\omega_{\ell}(t, x)= \pm 1$ means the direction of the $\ell$ th-transformation if $\mu_{\ell}(t, x) \neq$ 0 . Let us also notice that, at least if (3.10) is assumed, (3.8e) expresses, in particular, the balance of 
energy; indeed, by (3.4) we can calculate

$$
\begin{aligned}
\langle\mu, \omega\rangle_{\Omega_{T}} & =\left\langle\lambda \bullet \frac{\mathrm{d}}{\mathrm{d} t} \nu, \omega\right\rangle \\
& =\int_{0}^{T} \int_{\Omega} \sum_{\ell=1}^{L}\left|\lambda_{\ell} \bullet \frac{\mathrm{d} v}{\mathrm{~d} t}\right| \mathrm{d} x \mathrm{~d} t=\int_{\Omega} \sum_{\ell=1}^{L} \underset{t \in[0, T]}{\operatorname{Var}\left[\lambda_{\ell} \bullet \nu\right](t, x) \mathrm{d} x}
\end{aligned}
$$

if $\omega \in \Psi\left(\lambda \bullet \frac{\mathrm{d}}{\mathrm{d} t} \nu\right)$, as yielded by (3.12), where 'Var' denotes the total variation of a real-valued function on the interval $[0, T]$. Putting (3.13) into (3.11) then gives the desired energy balance:

$$
\begin{gathered}
\underbrace{V_{Q}^{\rho}\left(q_{0}\right)}_{\begin{array}{c}
\text { initial stored } \\
\text { energy }
\end{array}}-\underbrace{V_{Q}^{\rho}(q(T))}_{\begin{array}{c}
\text { final stored } \\
\text { energy }
\end{array}}+\underbrace{\int_{0}^{T}\left\langle F, \frac{\mathrm{d} q}{\mathrm{~d} t}\right\rangle \mathrm{d} t}_{\begin{array}{c}
\text { work made by } \\
\text { the external force }
\end{array}}=\mathcal{G}(0)-\mathcal{G}(T) \\
-\int_{0}^{T}\left\langle\frac{\mathrm{d} F}{\mathrm{~d} t}, q\right\rangle \mathrm{d} t=\langle\mu, \omega\rangle_{\Omega_{T}}=\int_{\Omega} \sum_{\ell=1}^{L} \operatorname{Var}_{t \in[0, T]}\left[P^{*} q(t)\right]_{\ell}(x) \mathrm{d} x .
\end{gathered}
$$

This last term in (3.14) thus shows how much energy is dissipated due to the phase transformation. Moreover, writing (3.12) with $v=0$, one gets

$$
\left\langle\frac{\mathrm{d} q}{\mathrm{~d} t}, P \omega\right\rangle=\max _{\xi \in \Psi(0)}\left\langle\frac{\mathrm{d} q}{\mathrm{~d} t}, P \xi\right\rangle,
$$

which is, in the context of classical plasticity, commonly called Hill's maximum-dissipation principle, see [13].

\subsection{Existence of weak solutions}

Existence of weak solutions will be proved rather constructively, by approximation by the Rothe method, i.e. by the semidiscretization in time by the implicit Euler formula using the partition of the interval $(0, T)$ with the constant time step $\tau>0$ (we assume $T / \tau$ integer):

$$
\partial R\left(\frac{q_{\tau}^{k}-q_{\tau}^{k-1}}{\tau}\right)+\partial V_{Q}^{\rho}\left(q_{\tau}^{k}\right) \ni F_{\tau}^{k}:=\frac{1}{\tau} \int_{(k-1) \tau}^{k \tau} F(t) \mathrm{d} t
$$

for $k=1,2, \ldots, T / \tau$ and for $q_{\tau}^{0}=q_{0}$. Furthermore, let us define $q_{\tau} \in C(0, T ; B)$ as piecewise affine interpolation (i.e. $\left.q_{\tau}(k \tau)=q_{\tau}^{k}\right)$ and $\bar{q}_{\tau} \in L^{\infty}(0, T ; B)$ piecewise constant interpolation, namely $\bar{q}_{\tau}(t)=q_{\tau}^{k}$ for $t \in((k-1) \tau, k \tau)$. Analogously, we define also $F_{\tau}$ and $\bar{F}_{\tau}$.

LEMMA 1 (Existence of approximate solutions.) For any $\tau>0$, there exists at least one $q_{\tau}$ defined above by the recursive formula (3.16), and this $q_{\tau}$ (and $\bar{q}_{\tau}$ ) fulfills, for suitable $\bar{\omega}_{\tau} \in L^{\infty}\left(\Omega_{T} ; \mathbb{R}^{L}\right)$ and $\bar{z}_{\tau} \in L^{\infty}\left(0, T ; B^{*}\right)$ piecewise constant on the considered partition of $[0, T]$ with the time step $\tau$, the following inequalities:

$$
\begin{aligned}
& P \bar{\omega}_{\tau}+\bar{z}_{\tau}=\bar{F}_{\tau}, \\
& \int_{0}^{T}\left\langle\bar{\omega}_{\tau}-\xi, P^{*} \frac{\mathrm{d} q_{\tau}}{\mathrm{d} t}-v\right\rangle \mathrm{d} t \geqslant 0 \quad \forall v \in L^{1}\left(\Omega_{T} ; \mathbb{R}^{L}\right), \xi \in L^{\infty}\left(\Omega_{T} ; \mathbb{R}^{L}\right), \quad \xi \in \partial \Psi(v), \\
& \int_{0}^{T}\left\langle\bar{z}_{\tau}-\xi, \bar{q}_{\tau}-v\right\rangle \mathrm{d} t \geqslant 0 \quad \forall v \in L^{1}(0, T ; B), \xi \in L^{\infty}\left(0, T ; B^{*}\right), \quad \xi \in \partial V_{Q}^{\rho}(v) .
\end{aligned}
$$


Proof. Existence of $q_{\tau}^{k}$ solving (3.16) can be proved by a direct method, relying on the fact that $q_{\tau}^{k}$ must solve the following minimization problem:

$$
\left.\begin{array}{ll}
\text { Minimize } & G_{\tau}^{k}(q):=\tau R\left(\frac{q-q_{\tau}^{k-1}}{\tau}\right)+V^{\rho}(q)-\left\langle F_{\tau}^{k}, q\right\rangle \\
\text { subject to } & q \in \bar{Q} .
\end{array}\right\}
$$

By the coercivity (2.2) with $p>1$, one can even show a non-concentration of the energy in the sense that even $q_{\tau}^{k} \in Q$, see (2.8).

Finally, the formula $\partial R=P \circ \partial \Psi \circ P^{*}$ holds because $\Psi$ is continuous in at least one point of Range $\left(P^{*}\right)$ (here even at all points), and therefore (3.16) just means $P \omega_{\tau}^{k}+z_{\tau}^{k}=F_{\tau}^{k}$ for some $\omega_{\tau}^{k} \in \partial \Psi\left(P^{*}\left(q_{\tau}^{k}-q_{\tau}^{k-1}\right) / \tau\right)$ and for some $z_{\tau}^{k} \in \partial V_{Q}^{\rho}\left(q_{\tau}^{k}\right)$. The former inclusion means just $\left\langle z_{\tau}^{k}-\right.$ $\tilde{\xi}, q_{\tau}^{k}-\tilde{v} \mid \geqslant 0$ for any $\tilde{v} \in B, \tilde{\xi} \in B^{*}, \tilde{\xi} \in \partial V_{Q}^{\rho}(\tilde{v})$, and in particular for $v=v(t)$ and $\xi=\xi(t)$ with $v$ and $\xi$ from (3.8f), $t \in((k-1) \tau, k \tau)$. The latter inclusion means $\left\langle\omega_{\tau}^{k}-\tilde{\xi}, P^{*}\left(q_{\tau}^{k}-q_{\tau}^{k-1}\right) / \tau-\tilde{v}\right\rangle \geqslant 0$ for any $\tilde{v} \in L^{1}\left(\Omega ; \mathbb{R}^{L}\right), \tilde{\xi} \in L^{\infty}\left(\Omega ; \mathbb{R}^{L}\right), \tilde{\xi} \in \partial \Psi(\tilde{v})$, and in particular for $\tilde{v}=v(t)$ and $\tilde{\xi}=\xi(t)$ with $v$ and $\xi$ from $(3.8 f), t \in((k-1) \tau, k \tau)$. By integration over the interval $((k-1) \tau, k \tau)$ and by summation for $k=1, \ldots, T / \tau$, one gets just (3.17a), (3.17b), (3.17c).

Lemma 2 (A priori estimates.) Under the assumptions (2.2), (2.13), (3.3), and (3.7a), (3.7b), (3.7c) the following estimates hold uniformly for all $\tau>0$ :

$$
\begin{aligned}
& \left\|q_{\tau}\right\|_{C(I, B)} \leqslant C_{1}, \\
& \left\|P^{*} q_{\tau}\right\|_{C\left(I, H^{1}\left(\Omega ; \mathbb{R}^{L}\right)\right)} \leqslant C_{2}=\mathcal{O}(\sqrt{\rho}), \quad \rho \text { from }(3.5), \\
& \left\|P^{*} \frac{\mathrm{d} q_{\tau}}{\mathrm{d} t}\right\|_{L^{1}\left(\Omega_{T} ; \mathbb{R}^{L}\right)} \leqslant C_{3}, \\
& \left\|\bar{\omega}_{\tau}\right\|_{L^{\infty}\left(\Omega_{T} ; \mathbb{R}^{L}\right)} \leqslant C_{4}, \\
& \left\|\bar{z}_{\tau}\right\|_{L^{\infty}\left(0, T, B^{*}\right)} \leqslant C_{5} .
\end{aligned}
$$

Proof. We want to test (3.16) by $q_{\tau}^{k}-q_{\tau}^{k-1}$. Having in mind (3.3), we use

$$
\begin{aligned}
\left\langle P \omega_{\tau}^{k}, \frac{q_{\tau}^{k}-q_{\tau}^{k-1}}{\tau}\right\rangle & =\left\langle\omega_{\tau}^{k}, P^{*} \frac{q_{\tau}^{k}-q_{\tau}^{k-1}}{\tau}\right\rangle \\
& =\Psi\left(P^{*} \frac{q_{\tau}^{k}-q_{\tau}^{k-1}}{\tau}\right)=\left\|P^{*} \frac{q_{\tau}^{k}-q_{\tau}^{k-1}}{\tau}\right\|_{L^{1}\left(\Omega ; \mathbb{R}_{1}^{L}\right)}
\end{aligned}
$$

for $\omega_{\tau}^{k} \in \partial \Psi\left(\tau^{-1} P^{*}\left(q_{\tau}^{k}-q_{\tau}^{k-1}\right)\right)$, which follows just by direct calculations if one realizes the explicit form of the subdifferential $\partial \Psi(v)=\left\{v^{*} \in L^{\infty}\left(\Omega ; \mathbb{R}_{\infty}^{L}\right) ; v^{*}(x)=v(x) /|v(x)|_{\infty}\right.$ if $v(x) \neq$ 0 or $\left|v^{*}(x)\right|_{\infty} \leqslant 1$ if $\left.v(x)=0\right\}$. Also, for $z_{\tau}^{k} \in \partial V_{Q}^{\rho}\left(q_{\tau}^{k}\right)$, we have

$$
\left\langle z_{\tau}^{k}, \frac{q_{\tau}^{k}-q_{\tau}^{k-1}}{\tau}\right\rangle \geqslant \frac{\mathrm{d}}{\mathrm{d} t} V_{Q}^{\rho}\left(q_{\tau}(t)\right) \geqslant \frac{V_{Q}^{\rho}\left(q_{\tau}^{k}\right)-V_{Q}^{\rho}\left(q_{\tau}^{k-1}\right)}{\tau}
$$


for a.a. $t \in((k-1) \tau, k \tau)$, which follows from the convexity of $V_{Q}^{\rho}$. Using (3.20), the first estimate in (3.21), and (3.7a), one gets for each time level $k$

$$
\begin{aligned}
\int_{0}^{k \tau} & \left\|P^{*} \frac{\mathrm{d} q_{\tau}}{\mathrm{d} t}\right\|_{L^{1}\left(\Omega ; \mathbb{R}_{1}^{L}\right)}+\frac{\mathrm{d}}{\mathrm{d} t} V_{Q^{\rho}}^{\rho}\left(q_{\tau}\right) \mathrm{d} t \leqslant \int_{0}^{k \tau}\left\langle P \bar{\omega}_{\tau}, \frac{\mathrm{d} q_{\tau}}{\mathrm{d} t}\right\rangle+\left\langle\bar{z}_{\tau}, \frac{\mathrm{d} q_{\tau}}{\mathrm{d} t}\right\rangle \mathrm{d} t \\
& =\int_{0}^{k \tau}\left\langle\bar{F}_{\tau}, \frac{\mathrm{d} q_{\tau}}{\mathrm{d} t}\right\rangle \mathrm{d} t=-\int_{\tau}^{k \tau}\left\langle\frac{\mathrm{d} F_{\tau}}{\mathrm{d} t}, \bar{q}_{\tau}\right\rangle \mathrm{d} t+\left\langle F_{\tau}^{k}, q_{\tau}^{k}\right\rangle-\left\langle F_{\tau}^{1}, q_{0}\right\rangle \\
& \leqslant \int_{\tau}^{k \tau}\left\|\frac{\mathrm{d} F_{\tau}}{\mathrm{d} t}\right\|_{B^{*}}\left\|q_{\tau}\right\|_{B} \mathrm{~d} t+\|F\|_{L^{\infty}\left(0, T ; B^{*}\right)}\left(\left\|q_{\tau}^{k}\right\|_{B}+\left\|q_{0}\right\|_{B}\right),
\end{aligned}
$$

where $F_{\tau} \in C\left(0, T ; B^{*}\right)$ denotes piece-wise affine interpolation of $\bar{F}_{\tau}$, which guarantees the discrete by-parts integration formula $\int_{0}^{k \tau}\left\langle\bar{F}_{\tau}, \frac{\mathrm{d}}{\mathrm{d} t} q_{\tau}\right\rangle \mathrm{d} t+\int_{\tau}^{k \tau}\left\langle\frac{\mathrm{d}}{\mathrm{d} t} F_{\tau}, \bar{q}_{\tau}\right\rangle \mathrm{d} t=\left\langle F_{\tau}^{k}, q_{\tau}^{k}\right\rangle-\left\langle F_{\tau}^{1}, q_{0}\right\rangle$. By (2.2) one has the coercivity $V_{Q}^{\rho}(q) \geqslant \varepsilon\|q\|_{B}^{p}-1 / \varepsilon$ for some 0 . Then, from the discrete Gronwall inequality with usage (3.7a) one gets $(3.19 a),(3.19 c)$. Then still $(3.19 b)$ follows by (3.5).

Moreover, from (3.3) and from the fact that $\bar{\omega}_{\tau} \in \partial \Psi\left(P^{*} \frac{\mathrm{d}}{\mathrm{d} t} q_{\tau}\right)$, we get immediately (3.19d) with $C_{4}=1$. From $\bar{z}_{\tau}=\bar{F}_{\tau}-P \bar{\omega}_{\tau}$ we then get still $(3.19 e)$ with $C_{5}=\|F\|_{L_{\mathrm{w}}^{\infty}\left(0, T ; B^{*}\right)}+$ $C_{4}\|P\|_{\mathcal{L}\left(L^{1}\left(\Omega ; \mathbb{R}_{1}^{L}\right), B^{\prime}\right)}$. In the case (3.3) and (2.11), we can even estimate $\|P\|_{\mathcal{L}\left(L^{1}\left(\Omega ; \mathbb{R}_{1}^{L}\right), B^{\prime}\right)}$ explicitly by $\max _{\ell=1}^{L}\left\|\lambda_{\ell}\right\|_{L^{\infty}\left(\Omega \times \mathbb{R}^{n}\right)}$.

LEMMA 3 (Limit passage.) Let (3.3) and (3.7a), (3.7b), (3.7c) hold. Then the sequence $\left\{q_{\tau}, P^{*} \frac{\mathrm{d} q_{\tau}}{\mathrm{d} t}, \bar{\omega}_{\tau}, \bar{z}_{\tau}\right\}_{\tau>0}$ has a weak* cluster point $(q, \mu, \omega, z)$ and there is a finer net (i.e. one must select it and re-index by a richer directed index set) such that

$$
\begin{array}{lc}
\bar{q}_{\tau} \rightarrow q & \left(\text { weakly*) in } L_{\mathrm{w}}^{\infty}(0, T ; B) \cong L^{1}\left(0, T ; B^{\prime}\right)^{*},\right. \\
P^{*} \frac{\mathrm{d} q_{\tau}}{\mathrm{d} t} \rightarrow \mu & \left(\text { weakly*) in } \operatorname{vba}\left(\Omega_{T} ; \mathbb{R}^{L}\right),\right. \\
\bar{\omega}_{\tau} \rightarrow \omega & \left(\text { weakly*) in } L^{\infty}\left(\Omega_{T} ; \mathbb{R}^{L}\right),\right. \\
P^{*} q_{\tau} \rightarrow P^{*} q & \left(\text { weakly*) in } L_{\mathrm{w}}^{\infty}\left(0, T ; H^{1}\left(\Omega ; \mathbb{R}^{L}\right)\right),\right. \\
P^{*} q_{\tau}(t) \rightarrow P^{*} q(t) \quad \text { in } L^{1}\left(\Omega ; \mathbb{R}^{L}\right) \text { for a.a. } t, \\
\bar{z}_{\tau} \rightarrow z & \left(\text { weakly*) in } L_{\mathrm{w}}^{\infty}\left(0, T ; B^{*}\right) .\right.
\end{array}
$$

Moreover, any $q$ obtained in this way is a weak solution to (3.1)-(3.2) with $\omega$ and $z$ obtained in (3.23c), (3.23f) just fitted for (3.8a) provided $T$ is chosen so that the second part of (3.8c) holds, which is a generic property with respect to $T$. If, in addition, $F \in L_{\mathrm{w}}^{\infty}\left(0, T ; B^{\prime}\right)$, then the regularity $z \in L_{\mathrm{w}}^{\infty}\left(0, T ; B^{\prime}\right)$ holds.

Proof. First, by the Banach theorem one can select a subsequence satisfying (3.23a), (3.23c), $(3.23 d)$, (3.23f). From (3.23d) and $(3.19 c)$ we get by Aubin's lemma, generalized for timederivatives bounded in $L^{1}$ by Dubinskiü [10] and Simon [39], the strong convergence $P^{*} q_{\tau} \rightarrow P^{*} q$ in $L^{1}\left(\Omega_{T} ; \mathbb{R}^{L}\right)$, or even in $L^{p}\left(0, T ; L^{1}\left(\Omega ; \mathbb{R}^{L}\right)\right)$ for any $p<+\infty$. Then one can additionally select a subsequence so that $(3.23 e)$ holds, too. As to $(3.23 b)$, one can still select a cluster point, using standard arguments by the Alaoglu-Bourbaki theorem for weak* relative compactness of bounded sets even in a nonmetrizable case; note that the predual to $\operatorname{vba}\left(\Omega_{T}\right) \cong L^{\infty}\left(\Omega_{T}\right)^{*}$ is not separable. 
Additionally, let us note that from the estimate

$$
\left\|P^{*} q_{\tau}-P^{*} \bar{q}_{\tau}\right\|_{L^{1}\left(\Omega ; \mathbb{R}_{1}^{L}\right)}=\frac{\tau}{2}\left\|P^{*} \frac{\mathrm{d} q_{\tau}}{\mathrm{d} t}\right\|_{L^{1}\left(\Omega ; \mathbb{R}_{1}^{L}\right)} \leqslant \frac{1}{2} C_{3} \tau
$$

one gets also $P^{*} \bar{q}_{\tau} \rightarrow P^{*} q$ strongly in $L^{1}\left(\Omega_{T} ; \mathbb{R}^{L}\right)$. Our aim is then to pass to the limit in (3.17a), $(3.17 b),(3.17 c)$.

Let us note that the nets $\left\{q_{\tau}\right\}_{\tau>0}$ and $\left\{\bar{q}_{\tau}\right\}_{\tau>0}$ have the same weak* limit because the difference $q_{\tau}-\bar{q}_{\tau}$ is eventually zero when tested by piecewise constant functions compatible with some partition of the interval $(0, T)$; of course, one must realize that such functions form a dense subset in $L^{1}\left(0, T ; B^{\prime}\right)$ provided $B^{\prime}$ is separable, as indeed assumed. Also, the second part of $(3.8 d)$ follows from the fact that, even for any $v \in W_{0}^{1,1}\left(0, T ; L^{\infty}\left(\Omega ; \mathbb{R}^{L}\right)\right)$, it holds that

$$
\begin{aligned}
\langle\mu, v\rangle_{\Omega_{T}} & =\left\langle\mathrm{w}^{*}-\lim _{\tau \rightarrow 0} P^{*} \frac{\mathrm{d} q_{\tau}}{\mathrm{d} t}, v\right\rangle=\lim _{\tau \rightarrow 0}\left\langle P^{*} \frac{\mathrm{d} q_{\tau}}{\mathrm{d} t}, v\right\rangle \\
& =\lim _{\tau \rightarrow 0}\left\langle\frac{\mathrm{d} q_{\tau}}{\mathrm{d} t}, P v\right\rangle=-\lim _{\tau \rightarrow 0}\left\langle q_{\tau}, \frac{\mathrm{d}}{\mathrm{d} t} P v\right\rangle \\
& =-\left\langle\mathrm{W}^{*}-\lim _{\tau \rightarrow 0} q_{\tau}, \frac{\mathrm{d}}{\mathrm{d} t} P v\right\rangle=-\left\langle q, \frac{\mathrm{d}}{\mathrm{d} t} P v\right\rangle \\
& =-\left\langle q, P \frac{\mathrm{d}}{\mathrm{d} t} v\right\rangle=-\left\langle P^{*} q, \frac{\mathrm{d}}{\mathrm{d} t} v\right\rangle ;
\end{aligned}
$$

here we have used integration by parts and the fact that $q_{\tau} \rightarrow q$ weakly* in $L_{\mathrm{w}}^{\infty}(0, T ; B)$.

Integrating (3.18) over $[0, T]$, one can see that $\bar{q}_{\tau}$ minimizes the functional $q \mapsto$ $\int_{0}^{T} V_{Q}^{\rho}(q(t)) \mathrm{d} t-\left\langle\bar{F}_{\tau}, q\right\rangle+\left\|P^{*} q-\Lambda_{\tau}\right\|_{L^{1}\left(\Omega_{T} ; \mathbb{R}^{L}\right)}$ with $\Lambda_{\tau}:=P^{*} \bar{q}_{\tau}(\cdot-\tau)$ over $q$ valued in $Q$ piecewise constant in time. Likewise for (3.24), we have also $\left\|\Lambda_{\tau}-P^{*} q_{\tau}\right\|_{L^{1}\left(\Omega_{T} ; \mathbb{R}^{L}\right)} \leqslant C_{3} \tau / 2$, hence, in fact, we have also proved $\Lambda_{\tau} \rightarrow \Lambda=P^{*} q$ in $L^{1}\left(\Omega_{T} ; \mathbb{R}^{L}\right)$, and thus in the limit $q$ minimizes the functional $q \mapsto \int_{0}^{T} V_{Q}^{\rho}(q(t)) \mathrm{d} t-\langle F, q\rangle+\left\|P^{*} q-\Lambda\right\|_{L^{1}\left(\Omega_{T} ; \mathbb{R}^{L}\right)}$. This implies that, for a.a. $t \in[0, T], q(t)$ minimizes $q \mapsto V_{Q}^{\rho}(q)-\langle F, q\rangle+\left\|P^{*} q-\Lambda\right\|_{L^{1}\left(\Omega ; \mathbb{R}^{L}\right)}$ with $F=F(t)$ and $\Lambda=\Lambda(t)$, we denote this minimum as $\mathcal{M}_{F, \Lambda}$. Let us emphasize that the value-function $(F, \Lambda) \mapsto \mathcal{M}_{F, \Lambda}: Q^{*} \times L^{1}\left(\Omega ; \mathbb{R}^{L}\right) \rightarrow \mathbb{R}$ is Lipschitz continuous. Moreover, as $\Lambda=P^{*} q$, it holds that $\mathcal{M}_{F(t), \Lambda(t)}=\mathcal{G}(t)$ for a.a. $t \in[0, T]$.

Let us take a countable collection of Borel subsets $O_{i} \subset \Omega$ such that $\operatorname{span}\left(\left\{\chi O_{i}\right\}_{i \in \mathbb{N}}\right)$ is dense in $L^{2}(\Omega)$. Denote the measure $\mu_{0}=\left.\mu\right|_{C\left(\bar{\Omega}_{T} ; \mathbb{R}^{L}\right)}$. The set functions $A \mapsto\left|\mu_{0}\right|\left(A \times O_{i}\right)$ are positive measures on $[0, T]$ with a bounded variation. By Lebesgue's decomposition, such measures are a.e. absolutely continuous, so let us consider a time where all these measures are absolutely continuous (which still holds for a.a. time instances due to the countability of this collection) and modify the problem by shifting the terminal time $T$ to this point. Moreover, by $(3.23 e)$ we can also assume that $T$ has been chosen so that both $P^{*} q_{\tau}(T) \rightarrow P^{*} q(T)$ and $\Lambda_{\tau}(T) \rightarrow \Lambda(T)$ in $L^{1}\left(\Omega ; \mathbb{R}^{L}\right)$. Thus we know that $t \mapsto \int_{O_{i}} \Lambda(t) \mathrm{d} x$ is continuous at $T$. By density of $\operatorname{span}\left(\left\{\chi O_{i}\right\}_{i \in \mathbb{N}}\right)$ in $L^{2}(\Omega)$, we can see that $t \mapsto \Lambda(t)$ is weakly continuous to $L^{2}\left(\Omega ; \mathbb{R}^{L}\right)$, hence in $L^{1}\left(\Omega ; \mathbb{R}^{L}\right)$, too. By $(3.19 b)$, this mapping is bounded even into $H^{1}\left(\Omega ; \mathbb{R}^{L}\right)$ hence, by compactness of the embedding $H^{1}(\Omega) \subset L^{2}(\Omega), t \mapsto \Lambda(t)$ is even norm continuous to $L^{1}\left(\Omega ; \mathbb{R}^{L}\right)$. Now, by the Lipschitz continuity of $(F, \Lambda) \mapsto \mathcal{M}_{F, \Lambda}$ and by the assumption $(3.7 b)$ and the special choice of $T$, $t \mapsto(F(t), \Lambda(t))$ is continuous at $T$, and so is $t \mapsto \mathcal{M}_{F(t), \Lambda(t)}$ at $t=T$. Hence the second part of $(3.8 c)$ is proved. 
Then we pass to the limit in $(3.17 a),(3.17 b),(3.17 c)$. From (3.17a) one gets immediately $P \omega+$ $z=F$ in $(3.8 d)$.

As we proved $P^{*} \bar{q}_{\tau} \rightarrow P^{*} q$ strongly in $L^{1}\left(\Omega_{T} ; \mathbb{R}^{L}\right)$, by (3.23c) one gets the convergence

$$
\begin{gathered}
\left\langle\bar{z}_{\tau}, \bar{q}_{\tau}\right\rangle=\left\langle\bar{F}_{\tau}, \bar{q}_{\tau}\right\rangle-\left\langle P \bar{\omega}_{\tau}, \bar{q}_{\tau}\right\rangle=\left\langle\bar{F}_{\tau}, \bar{q}_{\tau}\right\rangle-\left\langle\bar{\omega}_{\tau}, P^{*} \bar{q}_{\tau}\right\rangle \\
\rightarrow\langle F, q\rangle-\left\langle\omega, P^{*} q\right\rangle=\langle z, q\rangle,
\end{gathered}
$$

where we also used $\bar{F}_{\tau} \rightarrow F$ in $L^{\infty}\left(0, T ; B^{*}\right)$, which holds because of continuity of $F(\cdot)$. Thus one can pass to the limit in $(3.17 c)$, which proves (3.8f), thus $z \in \partial V_{Q}^{\rho}(q)$.

Furthermore, we have the estimate

$$
\begin{aligned}
\int_{0}^{T}\left\langle\bar{\omega}_{\tau}, P^{*} \frac{\mathrm{d} q_{\tau}}{\mathrm{d} t}\right\rangle \mathrm{d} t=\int_{0}^{T} & \left\langle P \bar{\omega}_{\tau}, \frac{\mathrm{d} q_{\tau}}{\mathrm{d} t}\right\rangle \mathrm{d} t=\int_{0}^{T}\left\langle\bar{F}_{\tau}-\bar{z}_{\tau}, \frac{\mathrm{d} q_{\tau}}{\mathrm{d} t}\right\rangle \mathrm{d} t \\
& \leqslant \int_{0}^{T}\left\langle\bar{F}_{\tau}, \frac{\mathrm{d} q_{\tau}}{\mathrm{d} t}\right\rangle \mathrm{d} t-V_{Q}^{\rho}\left(q_{\tau}(T)\right)+V_{Q}^{\rho}\left(q_{0}\right)
\end{aligned}
$$

where we used also

$$
\int_{0}^{T}\left\langle\bar{z}_{\tau}, \frac{\mathrm{d} q_{\tau}}{\mathrm{d} t}\right\rangle \mathrm{d} t=\sum_{k=1}^{T / \tau} z_{\tau}^{k}\left(q_{\tau}^{k}-q_{\tau}^{k-1}\right) \geqslant \sum_{k=1}^{T / \tau} V_{Q}^{\rho}\left(q_{\tau}^{k}\right)-V_{Q}^{\rho}\left(q_{\tau}^{k-1}\right)=V_{Q}^{\rho}\left(q_{\tau}(T)\right)-V_{Q}^{\rho}\left(q_{0}\right),
$$

see (3.21), with the convention that, if $T / \tau$ is not an integer, we interpolate in the last interval which is then made shorter. By (3.22), we get

$$
\begin{aligned}
\limsup _{\tau \rightarrow 0} \int_{0}^{T}\left\langle\bar{\omega}_{\tau}, P^{*} \frac{\mathrm{d} q_{\tau}}{\mathrm{d} t}\right\rangle \mathrm{d} t \leqslant \lim _{\tau \rightarrow 0}\left(\int_{0}^{T}\left\langle\bar{F}_{\tau}, \frac{\mathrm{d} q_{\tau}}{\mathrm{d} t}\right\rangle \mathrm{d} t-V_{Q}^{\rho}\left(q_{\tau}(T)\right)+V_{Q}^{\rho}\left(q_{0}\right)\right) \\
=\lim _{\tau \rightarrow 0}\left(-\int_{\tau}^{T}\left\langle\frac{\mathrm{d} F_{\tau}}{\mathrm{d} t}, \bar{q}_{\tau}\right\rangle \mathrm{d} t-V_{Q}^{\rho}\left(q_{\tau}(T)\right)\right. \\
\left.\quad+\left\langle F_{\tau}(T), q_{\tau}(T)\right\rangle+V_{Q}^{\rho}\left(q_{0}\right)-\left\langle F_{\tau}(\tau), q_{0}\right\rangle\right) \\
=-\int_{0}^{T}\left\langle\frac{\mathrm{d} F}{\mathrm{~d} t}, q\right\rangle \mathrm{d} t-\mathcal{G}(T)+\mathcal{G}(0)
\end{aligned}
$$

where we used also that $\lim _{\tau \rightarrow 0} V_{Q}^{\rho}\left(q_{\tau}(T)\right)-\left\langle F_{\tau}(T), q_{\tau}(T)\right\rangle=\lim _{\tau \rightarrow 0} \mathcal{M}_{F_{\tau}(T), \Lambda_{\tau}(T)}-\| \Lambda_{\tau}(T)-$ $P^{*} q_{\tau}(T) \|_{L^{1}\left(\Omega ; \mathbb{R}^{L}\right)}=\mathcal{M}_{F(T), \Lambda(T)}=\mathcal{G}(T)$ because $\lim _{\tau \rightarrow 0} \mathcal{M}_{F_{\tau}(T), \Lambda_{\tau}(T)}=\mathcal{M}_{F(T), \Lambda(T)}$ and $\lim _{\tau \rightarrow 0} \Lambda_{\tau}(T)=\Lambda(T)=P^{*} q(T)=\lim _{\tau \rightarrow 0} P^{*} q_{\tau}(T)$, and also $F_{\tau}(T) \rightarrow F(T)$ in $B^{*}$. Moreover, we used that $q_{\tau}(0)=q_{0}$ hence $V_{Q}^{\rho}\left(q_{0}\right)+\left\langle F_{\tau}(\tau), q_{0}\right\rangle \rightarrow \mathcal{G}(0)$. Also,

$$
\lim _{\tau \rightarrow 0} \int_{0}^{T}\left\langle\xi, P^{*} \frac{\mathrm{d} q_{\tau}}{\mathrm{d} t}\right\rangle \mathrm{d} t=\left\langle\mathrm{w}_{\tau \rightarrow 0}^{*-1 i m} P^{*} \frac{\mathrm{d} q_{\tau}}{\mathrm{d} t}, \xi\right\rangle=\langle\mu, \xi\rangle_{\Omega_{T}}
$$

with $\mu$ from (4.6). All this allows us to pass to the limit in (3.17b), which gives (3.8e).

Now, let us assume, without loss of generality, that $F(0)=0$; otherwise we can suitably modify $V$ simply by taking $V-F(0)$ instead. From (3.22) one gets, in particular, that $V_{Q}^{\rho}\left(q_{\tau}(t)\right) \leqslant V_{Q}^{\rho}\left(q_{0}\right)+$ $C t$ for a suitable $C<+\infty$. Hence, in the limit, $V_{Q}^{\rho}(q(t)) \leqslant V_{Q}^{\rho}\left(q_{0}\right)+C t$. From this, one can deduce $\limsup _{t \backslash 0} V_{Q}^{\rho}(q(t)) \leqslant V_{Q}^{\rho}\left(q_{0}\right)$. Also, $\langle F(t), q(t)\rangle \rightarrow\langle F(0), q(0)\rangle=0$ because $q(t)$ is bounded 
in $B$ while $\lim _{t \backslash 0} F(t)=F(0)=0$. Altogether, $\lim \sup _{t \backslash 0} \mathcal{G}(t) \leqslant \mathcal{G}(0)$. Yet, by $(3.7 c)$, one gets also $\lim \inf _{t \backslash 0} \mathcal{G}(t) \geqslant \mathcal{G}(0)$, proving thus the first part in $(3.8 c)$.

Finally, as $\omega \in L^{\infty}\left(\Omega_{T} ; \mathbb{R}^{L}\right)$, we have $P \omega \in L_{\mathrm{w}}^{\infty}\left(0, T ; B^{\prime}\right)$, thus also $z=F-P \omega$ belongs to this space provided $F$ does so, too.

Lemma 1 with Lemma 3 immediately leads to the following desired existence result.

Proposition 2 (Existence of weak solution.) Under the assumptions (3.3) and (3.7a), (3.7b), (3.7c) there is at least one weak solution $q \in L_{\mathrm{w}}^{\infty}(0, T ; B)$ to (3.1)-(3.2).

Note that one cannot similarly expect uniqueness of this weak solution because of the doublynonlinear structure (as pointed out already in [9]) and because the stationary inclusion (2.14) need not have a unique solution and $P^{*}$ may degenerate; even $P=0$ is admitted by the assumptions (3.7a), (3.7b), (3.7c). Nevertheless, one can possibly expect $P^{*} q(t)$ and $\bar{V}(q(t))$ to be determined uniquely and a rigorous proof would justify our definition of the weak solution, but this point seems difficult

Let us also remark that the directed index set mentioned in Lemma 3 can be always taken in an explicit way, namely as the set of all finite subsets of $L^{\infty}\left(\Omega_{T} ; \mathbb{R}^{L}\right)$ directed by the inclusion.

\subsection{Remarks on steady states}

Assuming $F$ constant in time, one can naturally ask about steady states of (3.1) (or equally of (2.21)). It is an interesting observation that they need not solve (2.14), i.e. they need not to minimize $V-F$ on $Q$. In accord with the standard definition, we call $q^{*}$ weakly Pareto optimal with respect to a collection of criteria $\left\{F_{l}: B \rightarrow \mathbb{R} \cup+\infty\right\}_{\iota \in I}$ if $\forall q \in B \exists \iota \in I: F_{\iota}\left(q^{*}\right) \leqslant F_{l}(q)$. Sometimes, this mode of multicriteria optimality is also called the Slater optimal.

Proposition 3 (Optimality of steady states.) Any steady state $q^{*}$ of (3.1) is weakly Pareto optimal with respect to the collection of criteria $\left\{V_{Q}^{\rho}+P \omega-F\right\}_{\omega \in \partial \Psi(0)}$.

Proof. That $q^{*}$ is a steady state just means that $\partial \bar{V}_{Q}^{\rho}\left(q^{*}\right)+P \omega=F$ for some $\omega \in \partial \Psi\left(P^{*} \frac{\mathrm{d}}{\mathrm{d} t} q^{*}\right)=$ $\partial \Psi\left(P^{*} 0\right)=\partial \Psi(0)$. In view of the convexity of $V_{Q}^{\rho}$, this further just means that $q^{*}$ minimizes $V_{Q}^{\rho}+P \omega-F$ and therefore $q^{*}$ is certainly weakly Pareto optimal with respect to any set of criteria which contains $V_{Q}^{\rho}+P \omega-F$.

Unfortunately, the converse implication does not hold because $\partial \Psi\left(P^{*} \frac{\mathrm{d}}{\mathrm{d} t} q\right) \subset \partial \Psi(0)$ need not imply $P^{*} \frac{\mathrm{d}}{\mathrm{d} t} q=0$.

From the proof it is also clear that Proposition 3 does not, in fact, say much. Yet, it indicates the functionals which $q^{*}$ may minimize, and in particular shows that this set may be larger than only a single functional $V_{O}^{\rho}-F$.

Another interesting observation is that some regularization $\Psi_{\varepsilon}$ of $\Psi$ near 0 may completely change the structure of steady states: if $\partial \Psi_{\varepsilon}=\{0\}$ (see also (2.23)), then Proposition 3 says that $q^{*}$ does minimize the original 'elastic' part $\bar{V}_{Q}^{\rho}-F$, i.e. the stored energy with the potential energy of the support and external body forces. This observation may be interpreted as identifying the validity range of models relying on the minimum-energy principle like $[2,3,5,23]$. A related question in this context is whether, in this regularized case, $q(T)$ minimizes $V_{Q}^{\rho}-F$ for $T \rightarrow \infty$. The affirmative answer can be proved by only slight modification of the standard procedure; for a very special 
case $R(\dot{q})=\frac{1}{2}\|\dot{q}\|_{B}^{2}$ and $B$ a Hilbert space we refer to Aubin and Cellina [1: p.160]. We need to prolong the solution for $T \rightarrow \infty$ : Having a weak solution up to a time $T=T_{1}$, we can continue it on a suitable interval $\left[T_{1}, T_{2}\right]$ (of nondegenerating length) with the initial condition $q\left(T_{1}\right)$. The inequalities (3.8e) and (3.8f) can be then summed easily, obtaining $\int_{0}^{T_{1}+T_{2}}\langle\xi-\omega, v\rangle-\left\langle\frac{\mathrm{d}}{\mathrm{d} t} F, q\right\rangle \mathrm{d} t-$ $\langle\mu, \xi\rangle_{\Omega_{\left(T_{1}+T_{2}\right)}} \geqslant \mathcal{G}\left(T_{1}+T_{2}\right)-\mathcal{G}(0)$ and $\int_{0}^{T_{1}+T_{2}}\langle z-\xi, q-v\rangle \mathrm{d} t \geqslant 0$, respectively. By continuation, we can get $q$ defined a.e. on $[0,+\infty)$. A regularization $\Psi_{\varepsilon}$ for $\Psi=\|\cdot\|_{L^{1}\left(\Omega ; \mathbb{R}^{L}\right)}$ can be, for example $\Psi_{\varepsilon}(\zeta)=\int_{\Omega} \sqrt{|\zeta(x)|^{2}+\varepsilon} \mathrm{d} x$; then $\Psi_{\varepsilon}$ is even differentiable and satisfies

$$
\left\|\Psi_{\varepsilon}^{\prime}(\zeta)\right\|_{L^{2}\left(\Omega ; \mathbb{R}_{2}^{L}\right)}^{2}=\int_{\Omega} \frac{\zeta^{2}(x)}{\zeta^{2}(x)+\varepsilon} \mathrm{d} x \leqslant \frac{1}{\varepsilon} \int_{\Omega} \frac{\zeta^{2}(x)}{\sqrt{\zeta^{2}(x)+\varepsilon}} \mathrm{d} x=\left\langle\Psi_{\varepsilon}^{\prime}(\zeta), \zeta\right\rangle .
$$

PROPOSITION 4 (Asymptotical behaviour of energy.)) Let $F$ be constant in time, the regularized potential of dissipative forces $\Psi_{\varepsilon}$ be given by $\Psi_{\varepsilon}(\zeta)=\int_{\Omega} \sqrt{|\zeta(x)|^{2}+\varepsilon} \mathrm{d} x$, and suppose that the dissipated energy does not concentrate so that the weak solution $q$ of (3.1)-(3.2) (with $\Psi_{\varepsilon}$ instead of $\Psi$ ) can be prolonged on $[0,+\infty)$ as described above. Then $q(T)$ minimizes $V_{Q}^{\rho}-F$ for $T \rightarrow \infty$ in the sense that

$$
\lim _{T \rightarrow+\infty} \operatorname{essinf}_{t \in[0, T]}\left[V_{Q}^{\rho}-F\right](q(t))=\min \left(V_{Q}^{\rho}-F\right) .
$$

Proof. First, one can consider $F=0$ without any loss of generality. Let us prove that the dissipation rate $\left\langle\Psi_{\varepsilon}^{\prime}\left(P^{*} \frac{\mathrm{d}}{\mathrm{d} t} q\right), P^{*} \frac{\mathrm{d}}{\mathrm{d} t} q\right\rangle$ can be made arbitrarily small for $t$ large, i.e.

$$
\forall \delta>0 \quad \exists I_{\delta} \subset \mathbb{R}^{+}, \operatorname{meas}\left(I_{\delta}\right)=+\infty, \quad \forall t \in I_{\delta}:\left\langle\Psi_{\varepsilon}^{\prime}\left(P^{*} \frac{\mathrm{d}}{\mathrm{d} t} q\right), P^{*} \frac{\mathrm{d}}{\mathrm{d} t} q\right\rangle \leqslant \delta .
$$

Let us set

$$
I_{\delta}:=\left\{t \in[0,+\infty) ; \quad\left\langle\Psi_{\varepsilon}^{\prime}\left(P^{*} \frac{\mathrm{d}}{\mathrm{d} t} q\right), P^{*} \frac{\mathrm{d}}{\mathrm{d} t} q\right\rangle \leqslant \delta\right\} .
$$

As $\mu$ has a density, we have at our disposal the energy balance (3.14) modified for $F=0$ but with the more general (but smooth) $\Psi_{\varepsilon}$ used here, i.e. $V_{Q}^{\rho}(q(T))-V_{Q}^{\rho}\left(q_{0}\right)=-\int_{0}^{T}\left\langle\Psi_{\varepsilon}^{\prime}\left(P^{*} \frac{\mathrm{d} q}{\mathrm{~d} t}\right), P^{*} \frac{\mathrm{d} q}{\mathrm{~d} t}\right\rangle$. This gives

$$
\begin{aligned}
& V_{Q}^{\rho}\left(q_{0}\right)=V_{Q}^{\rho}(q(T))+\int_{0}^{T}\left\langle\Psi_{\varepsilon}^{\prime}\left(P^{*} \frac{\mathrm{d} q}{\mathrm{~d} t}\right), P^{*} \frac{\mathrm{d} q}{\mathrm{~d} t}\right\rangle \mathrm{d} t \\
& \geqslant V_{Q}^{\rho}(q(T))+\int_{[0, T] \backslash I_{\delta}}\left\langle\Psi_{\varepsilon}^{\prime}\left(P^{*} \frac{\mathrm{d} q}{\mathrm{~d} t}\right), P^{*} \frac{\mathrm{d} q}{\mathrm{~d} t}\right\rangle \mathrm{d} t \geqslant V_{Q}^{\rho}(q(T))+\delta \operatorname{meas}\left(\mathbb{R}^{+} \backslash I_{\delta}\right) .
\end{aligned}
$$

If meas $\left(I_{\delta}\right)=+\infty$, the last term would equal $+\infty$, which is not possible since $V_{Q}^{\rho}(q(T))$ is bounded from below and $V_{Q}^{\rho}\left(q_{0}\right)<+\infty$. Thus, (3.30) is proved.

Then, for a.a. $t \in I_{\delta}$,

$$
\begin{aligned}
\underset{\tau>0}{\operatorname{essinf}} V_{Q}^{\rho}(q(\tau)) & \leqslant V_{Q}^{\rho}(q(t)) \leqslant V_{Q}^{\rho}(\tilde{q})+\langle z(t), q(t)-\tilde{y}\rangle \\
& =V_{Q}^{\rho}(\tilde{q})+\langle P \omega(t), q(t)-\tilde{y}\rangle \\
& \leqslant V_{Q}^{\rho}(\tilde{q})+\|P\|_{\mathcal{L}\left(L^{2}\left(\Omega ; \mathbb{R}_{2}^{L}\right), B^{\prime}\right)}\|\omega(t)\|_{L^{2}\left(\Omega ; \mathbb{R}_{2}^{L}\right)}\|q(t)-\tilde{y}\|_{B}
\end{aligned}
$$


for $z=P \omega$ and $\omega=\Psi_{\varepsilon}^{\prime}\left(P^{*} \frac{\mathrm{d}}{\mathrm{d} t} q\right)$; note that, in the case (3.3) with (2.1), the norm $\|P\|_{\mathcal{L}\left(L^{2}\left(\Omega ; \mathbb{R}_{2}^{L}\right), B^{\prime}\right)}$ can be estimated by $N \max _{\ell=1}^{L}\left\|\lambda_{\ell}\right\|_{L^{\infty}\left(\Omega \times \mathbb{R}^{n}\right)}$ with $N$ denoting the norm of the embedding $L^{2}\left(\Omega ; \mathbb{R}_{2}^{L}\right) \subset L^{1}\left(\Omega ; \mathbb{R}_{1}^{L}\right)$. By using (3.28) with (3.30), $\|\omega(t)\|$ can be made arbitrarily small at least for some $t$. This shows that $\inf _{\tau>0} V_{Q}^{\rho}(q(\tau)) \leqslant V_{Q}^{\rho}(\tilde{q})$. As $\tilde{q} \in Q$ is arbitrary, we eventually get (3.29).

Let us notice that, for the rate-independent model which must have the dissipative potential nondifferentiable at 0 , any analogue to (3.28) cannot hold and the proof breaks at the point that $\omega(t)$ cannot be made small.

\section{Example: a double-well problem}

We also want to demonstrate the potential applicability of the model computationally, focusing on the 'double-well' situation indicated of Figs 1 and 2. In this case, one expects so-called quasiplasticity effects. To model just material properties and to suppress the influence of the particular geometry of a specimen, we focus on the 'zero-dimensional' case, i.e. we take not only $n=1$ but additionally assume $v_{x}$ independent of $x$ and hence $u(x)$ affine. In other words, we consider a small specimen so that any spatial dependence can be neglected. This makes implementation of the model easy and simultaneously illustrative. Moreover, this automatically allows us to put off the regularization by $\rho$ that we had to make artificially for the sake of rigorous analysis.

\subsection{Data}

As we omit any dependence on $x$, we consider $u$ as a scalar parameter, and then the strain simply as $e=u$ (which corresponds to a unit length of the homogeneously deformed specimen), and $v=v_{x}$. Furthermore, we take $\varphi, \psi$ and $f$ defined by

$$
\begin{aligned}
& \varphi(u, e):=\frac{1}{2} \min \left(E_{\alpha}\left(e-e_{\alpha}\right)^{2}, E_{\beta}\left(e-e_{\beta}\right)^{2}\right) \\
& \psi(u):=K u^{2}, \\
& \langle f(t), u\rangle=2 K z(t) u,
\end{aligned}
$$

where the Young moduli $E_{\alpha}=E_{\beta}=15$ for our calculations and $e_{\alpha}=-e_{\beta}=1$ so that the doublewell potential is symmetric, having two minima for the strains $e= \pm 1$ as in Fig. 2(a); $z=z(t)$ is an external loading varying in time, and $K=10$ characterizes the spring, see Fig. 1(a). Up to a constant, $(4.1 b),(4.1 c)$ yields the energy of this 'virtual' spring as $K(u-z(t))^{2}$, which gives the force (being simultaneously the stress $\sigma$ in the specimen) equal to

$$
f=\sigma=2 K(u-z(t)) .
$$

A dissipative mechanism related with the phase transition can be described on the mesoscopical level if the phase indicator $\lambda_{\ell}$ is taken constant on particular phases, i.e. we use (2.16 with $L=1$ and, denoting $\lambda=\lambda_{1}$,

$$
\lambda(e)= \begin{cases}0 & \text { for } e<-\zeta, \\ \frac{e+\zeta}{2 \zeta} \mathcal{E}_{\alpha \beta} & \text { for } e \in[-\zeta, \zeta], \\ \mathcal{E}_{\alpha \beta} & \text { for } e>\zeta,\end{cases}
$$


where $\mathcal{E}_{\alpha \beta}=4$ is an energy dissipated within phase transformation per unit volume, and $\zeta>0$ is some small regularizing parameter to make the phase indicator $\lambda$ continuous also in the metastable region, without actual influence on computation, see Fig. 2(b).

At the initial time $t=0$, we begin with a stress-free $50 / 50 \%$ mixture of the two phases, i.e. we set up the initial conditions as

$$
u_{0}=0, \quad\left[v_{0}\right]_{x}=\frac{1}{2} \delta_{-1}+\frac{1}{2} \delta_{1},
$$

where $\delta_{e}$ denotes the Dirac measure supported at the strain $e$; note that the initial impulse $p_{0}$ is not relevant since $T_{\text {kin }} \equiv 0$ is assumed.

\subsection{Simulation of cyclical loading response}

The formula (3.14), or rather its discrete version (3.22), suggests a numerical check the energy balance. Here, at a current level $l$, one has the work of the external force

$$
W_{\tau}^{l}:=\int_{0}^{l \tau} f_{\tau}(t) \frac{\mathrm{d} u_{\tau}}{\mathrm{d} t} \mathrm{~d} t=2 K \tau \sum_{k=1}^{l}\left(\frac{u_{\tau}^{k}+u_{\tau}^{k-1}}{2}-\frac{z_{\tau}^{k}+z_{\tau}^{k-1}}{2}\right) \cdot \frac{u_{\tau}^{k}-u_{\tau}^{k-1}}{\tau}
$$

and the energy dissipated during phase transformation equals

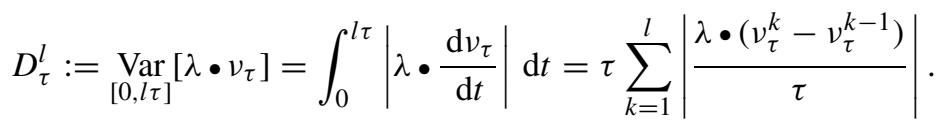

Recall that 'Var' denotes the total variations on the time interval indicated. Altogether, the expected total energy balance says that

$$
\varphi \bullet v_{\tau}^{k}+D_{\tau}^{k}+W_{\tau}^{k} \sim \varphi \bullet \nu_{\tau}^{0}=0 ;
$$

to be more specific, (3.14) gives only equality, but the numerical experiments suggest rather that equality approximately holds. The calculated difference is due to numerical dissipation through time discretization as well as the discretization of the Young measure by (4.10) below, and possibly also due to the regularization of the singular stiffness matrix in (4.14) as well as round-off errors. Actually, one can easily see by numerical experiments that pushing $\tau$ to zero as well as increasing the number of atoms in (4.10) makes the error in (4.7) smaller.

A response of the strain $e=\nabla u$, the stress $\sigma$, and the volume fraction $\lambda \bullet v$ within cyclical loading $z$ is displayed in Figs 3 and 4. The former figure shows a periodic response to periodic loading, which reflects a perfect reversibility of martensitic transformation.

The last trace in Fig. 3 shows the difference in (4.7) relative to the total energy exchanged, i.e.

$$
\text { relative error in energy balance }:=\frac{\varphi \bullet v_{\tau}^{k}+D_{\tau}^{k}+W_{\tau}^{k}}{\varphi \bullet v_{\tau}^{k}+D_{\tau}^{k}+\left|W_{\tau}^{k}\right|}
$$

giving thus a rough a posteriori idea about the precision of the presented calculations. In fact, the first four traces as well as Figs 4-6 are practically independent of $\tau>0$. Contrary to this, the last one is, of course, very dependent on $\tau$ as well as on $J$ (see Section 4.3) and Fig. 3 displays a 


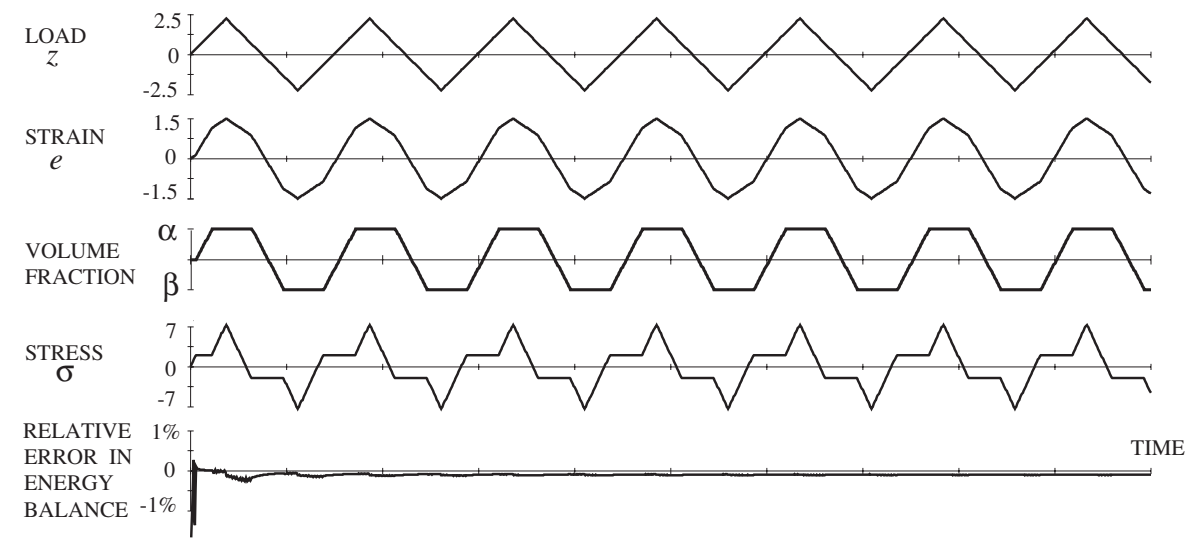

FIG. 3. Cyclical loading and the responses as functions of time.
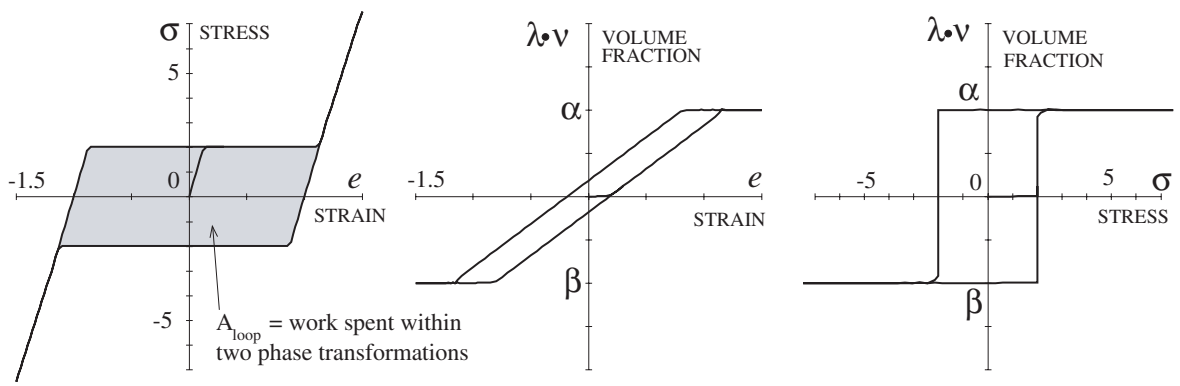

FIG. 4. Hysteresis effects corresponding to Fig. 3.

particular case of $\tau$ yielding about 220 time steps per one period and the relative error (4.8) of order $10^{-3}$ for large time; rather large values of this relative error at the beginning are not significant, being caused by the small values taken by the denominator in (4.8).

When drawing the stress/strain diagram (leftmost in Fig. 4) one can observe hysteresis effects similar to plasticity, called quasiplasticity. The area of the hysteresis loop $A_{\text {loop }}$ is proportional to the energy dissipated within phase transformation. Within one loop, a particular phase is transformed to the other (dissipating just the energy $\mathcal{E}_{\alpha \beta}$ ) and back (again dissipating the same amount of energy $\left.\mathcal{E}_{\alpha \beta}\right)$, henceforth

$$
A_{\text {loop }}=2 \mathcal{E}_{\alpha \beta}
$$

and indeed the area of the stress/strain loop (grey in Fig. 4) is $A_{\text {loop }}=2 \mathcal{E}_{\alpha \beta}=8$ with a precision beyond visibility even under great magnification. Contrary to classical plasticity, the quasiplasticity is a reversible process, which is also seen from Fig. 4 as the loops do not depend on the number of cycles.

Let us emphasize that this model is numerically stable, as also reflects the rigorous analysis from Section 3.2. One can easily play with it, varying the parameters involved. To illustrate the influence of the change of the potential $\varphi$, let us perturb the right-hand well vertically, as shown in Fig. 5 (left), the remaining drawings there displaying the corresponding response. 

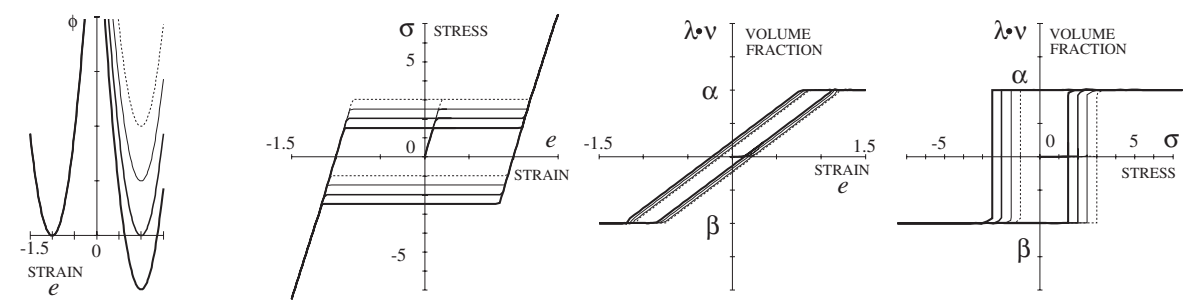

FIG. 5. Influence of the vertical variation of right-hand potential well.
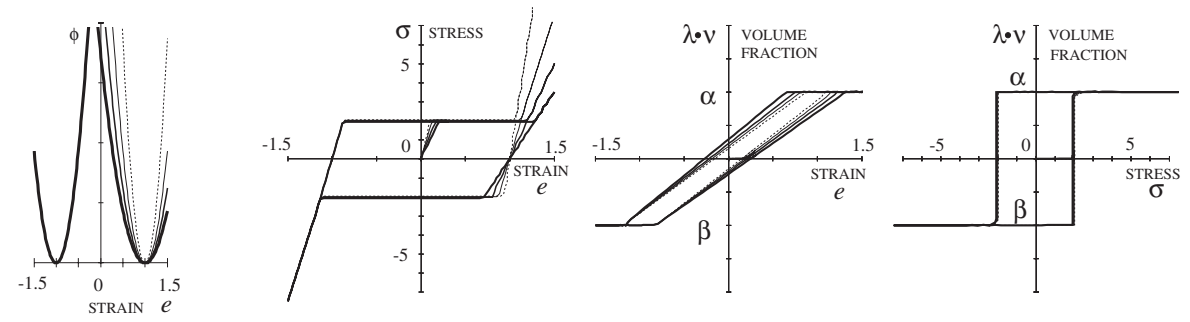

FIG. 6. Influence of the stiffness variation of right-hand potential well.

Another illustrative variation consists in playing with the stiffness of the right-hand phase, i.e. we keep $E_{\alpha}=15$ and vary successively $E_{\beta}=7,10,15$, and 30 (Fig. 6). One can, of course, see that the area of the stress/strain hysteresis loop, being determined by (4.9), indeed does not change within all these perturbations.

Let us conclude by stating that the above results agrees to a large extent with experiments performed on low-temperature martensitic structures cyclically loaded: see, for example, $[12,16,17]$. The parameters of our model, i.e. the strain of unloaded phases $e_{\alpha}$ and $e_{\beta}$, the energy $\mathcal{E}_{\alpha \beta}$ dissipated within the phase transformation, and the Young moduli of particular phases $E_{\alpha}$ and $E_{\beta}$, can be read from the stress/strain diagram which usually results experimentally by measurement, assuming that a single-crystal uniaxial-loading experiment like that of Fig. 1(a) can be arranged.

\subsection{Remarks on numerical implementation}

The inclusion (3.1) to be solved at each time level $k$ involves the Young measure $v^{k}$ appearing in each $q_{\tau}^{k}=\left(u^{k}, v^{k}\right)$. Even if considered homogeneous (i.e. $x$-independent), the probability measure $v_{x}^{k}=v^{k}$ cannot, in general, be implemented on computers and must also be discretized. Here we employ the discretization with fixed set $S=\left\{e_{j}\right\}_{j=1}^{J} \subset \mathbb{R}$ of $J$ atoms as in [32], which leads to a minimization of $G_{\tau}^{k}$ from (3.18) over the set

$$
\begin{aligned}
Q_{S} & :=\left\{(u, v) \in Q ; v=\sum_{j=1}^{J} \gamma_{j} \delta_{e_{j}}\right\} \\
& =\left\{(u, v) ; v=\sum_{j=1}^{J} \gamma_{j} \delta_{e_{j}}, \gamma_{j} \geqslant 0, \sum_{j=1}^{J} \gamma_{j}=1, \sum_{j=1}^{J} \gamma_{j} e_{j}=\frac{u}{l}\right\},
\end{aligned}
$$


where $l=1$ is the length of the specimen. In view of (4.10), $v \in Q_{S}$ will be represented here (and also in a computer) as a vector $\gamma \in \mathbb{R}^{J}$, which we will record by writing $v \cong \gamma$ for $v \in$ $Q_{S}$. Note that $Q_{S}$ is always a convex subset of $Q$, or (adapting this representation) of $\mathbb{R}^{J}$. If the dissipative potential $R$ were quadratic, this discretization of (3.18) would lead to a linear-quadratic program to be solved at each time level $k$. For $R$ from (2.16) we get, however, a nonsmooth convex program which is much more difficult in general. Fortunately, the minimized functional $G_{\tau}^{k}$ is a sum $G_{\tau, 1}^{k}+G_{\tau, 2}^{k}$ of a convex quadratic function $G_{\tau, 1}^{k}$ and a convex nonsmooth function $G_{\tau, 2}^{k}$ whose graph is polyhedral, namely

$$
\begin{aligned}
& G_{\tau, 1}^{k}(q):=K(u-z(k \tau))^{2}+\sum_{j=1}^{J} \gamma_{j} \varphi\left(e_{j}\right), \quad \text { with } \varphi(x, u, e)=\varphi(e), \\
& G_{\tau, 2}^{k}(q):=\left|\lambda \bullet v^{k-1}-\sum_{j=1}^{J} \gamma_{j} \lambda\left(e_{j}\right)\right|, \quad \lambda \bullet v^{k-1}=\sum_{j=1}^{J} \gamma_{j}^{k-1} \lambda\left(e_{j}\right),
\end{aligned}
$$

where $\left(u^{k-1}, b^{k-1}\right)$ denotes the solution from the level $k-1$. If a quadratic dissipation potential or the kinetic energy (2.20) were involved in our model, some additional quadratic contributions would appear in $G_{\tau, 1}^{k}$.

It is certainly worth adding one variable more and transforming the problem of minimization of $G_{\tau}^{k}$ over $Q_{S}$ to the following problem:

$$
\left.\begin{array}{cl}
\text { Minimize } & G_{\tau, 1}^{k}(q)+a \\
\text { subject to } & G_{\tau, 2}^{k}(q) \leqslant a, \\
& q \in Q_{S}, \quad a \in \mathbb{R},
\end{array}\right\}
$$

which leads to a linear-quadratic program to be solved at each time level $k$.

Lemma 4 If $(q, a) \cong(u, \gamma, a)$ solves (4.13), then $a=G_{\tau, 2}^{k}(q)$ and $q$ minimizes $G_{\tau}^{k}$ over $Q_{S}$. Conversely, if $q$ minimizes $G_{\tau}^{k}$ over $Q_{S}$, then the pair $\left(q, G_{\tau, 2}^{k}(q)\right)$ solves (4.13).

Proof. Take $(q, a) \in Q_{S} \times \mathbb{R}$ a solution of (4.13). In particular, $G_{\tau, 2}^{k}(q) \leqslant a$. Supposing $G_{\tau, 2}^{k}(q)<$ $a$, we can find that $\left(q, G_{\tau, 2}^{k}(q)\right)$ is admissible for (4.13) and gives a lower cost, contradicting thus the optimality of $(q, a)$. Henceforth, necessarily $a=G_{\tau, 2}^{k}(q)$. Then $G_{\tau, 1}^{k}(q)+a=G_{\tau}^{k}(q) \leqslant$ $G_{\tau, 1}^{k}(\tilde{q})+\tilde{a}$ for any $(\tilde{q}, \tilde{a}) \in Q_{S} \times \mathbb{R}$ with $G_{\tau, 2}^{k}(\tilde{q}) \leqslant \tilde{a}$, in particular also for $\left(\tilde{q}, G_{\tau, 2}^{k}(\tilde{q})\right)$ with any $\tilde{q} \in Q_{S}$. This just shows that $q$ minimizes $G_{\tau}^{k}$ over $Q_{S}$.

Conversely, take $q \in Q_{S}$ such that $G_{\tau, 1}^{k}(q)+G_{\tau, 2}^{k}(q) \leqslant G_{\tau, 1}^{k}(\tilde{q})+G_{\tau, 2}^{k}(\tilde{q})$ for any $\tilde{q} \in$ $Q_{S}$. Then $(q, a):=\left(q, G_{\tau, 2}^{k}(q)\right)$ is certainly admissible for (4.13) and, moreover, $G_{\tau, 1}^{k}(q)+a=$ $G_{\tau}^{k}(q) \leqslant G_{\tau}^{k}(\tilde{q})=G_{\tau, 1}^{k}(\tilde{q})+G_{\tau, 2}^{k}(\tilde{q}) \leqslant G_{\tau, 1}^{k}(\tilde{q})+\tilde{a}$ for any $(\tilde{q}, \tilde{a})$ admissible for (4.13). This shows that $(q, a)$ solves (4.13). 
Then, at the time level $k,(4.13)$ leads to the linear-quadratic program

$$
\left.\begin{array}{ll}
\text { Minimize } & \sum_{j=1}^{J} \gamma_{j} \varphi\left(e_{j}\right)+K\left(u-z^{k}\right)^{2}+a \\
\text { subject to } & \sum_{j=1}^{J} \gamma_{j}=1, \quad a+\sum_{j=1}^{J} \gamma_{j} \lambda\left(e_{j}\right) \geqslant \lambda \bullet v_{\tau}^{k-1}, \\
& \sum_{j=1}^{J} \gamma_{j} e_{j}=\frac{u}{l}, \quad a-\sum_{j=1}^{J} \gamma_{j} \lambda\left(e_{j}\right) \geqslant-\lambda \bullet v_{\tau}^{k-1}, \\
& (u, \gamma, a) \in \mathbb{R} \times \mathbb{R}^{J} \times \mathbb{R}, \quad \gamma_{j} \geqslant 0 \text { for } 1 \leqslant j \leqslant J,
\end{array}\right\}
$$

where $z^{k}=z(k \tau)$. For our calculations, we used $J=148$ fixed atoms $S=\left\{e_{j}\right\}_{j=1}^{148}$ supported on the effective interval $[-1.5,+1.5]$, see Fig. 2(a). Thus (4.13) is a linear-quadratic program on $\mathbb{R}^{1+J+1}=\mathbb{R}^{150}$ with two equality constraints, two inequality constraints, and 148 box constraints. This program was solved (after a numerical regularization by adding $10^{-6}$ into the diagonal of the matrix of the minimized quadratic form) by Schittkowski's implementation of Powell's algorithm [25].

\subsection{Additional remarks}

The form of the dissipative potential $R$ obviously plays the key role and more sophisticated forms possibly also justified theoretically would probably explain finer effects observed experimentally. Moreover, the uniaxial deformation according Fig. 1 usually cannot be realized, the actual situation being fully three-dimensional; see also the numerical two-dimensional experiments in [35].

The potential $\varphi$ often heavily depends on temperature, usually changing even the number of the wells and creating characteristic shape-memory effects. Typically, when increasing temperature, the quasiplasticity presented here gradually becomes a so-called pseudoelasticity. The non-isothermal variant of the above model has been developed [34] and some numerical experiments also done, but a rigorous analysis of this generalization seems difficult.

Also, a study of the hysteretic effects as in Figs 4-6 in the context of a general theory of hysteresis seem worthwhile, see Krejčí [18] for a survey showing intimate connections with the model of type (3.1).

\section{Acknowledgements}

The author is grateful to Dr. A. R. Srinivasa for his remark [40] about positively homogeneous dissipative potentials and also to Professor K. R. Rajagopal, for careful reading of the manuscript and for many remarks, as well as for discussions during its preparation. In addition, many comments of the four anonymous referees are acknowledged. This research has been supported by the NSF/Czech grant no. 2996K1020 during the author's visit at Texas A \& M University and partly also by the grants 201/00/0768 (GA ČR), A 1075005 (GA AV ČR), and MSM 11320007 (MŠMT ČR).

\section{REFERENCES}

1. Aubin, J.-P. \& Cellina, A. Differential Inclusions. Wiley, New York (1984). 
2. Ball, J. M. \& James, R. D. Fine phase mixtures as minimizers of energy. Archive Rat. Mech. Anal. 100, (1988) 13-52.

3. BALL, J. M. \& JAMES, R. D. Proposed experimental tests of a theory of fine microstructure and the two-well problem. Phil. Trans. Royal Soc. London A 338, (1992) 389-450.

4. Bedford, A. Hamilton's Principle in Continuum Mechanics. Pitman, Boston, MA, (1985).

5. BHATTACHARYA, K. Comparison of geometrically nonlinear and linear theories of martensitic transformation. Cont. Mech. Thermodyn. 5, (1993) 205-242.

6. BréZIS, H. Operateurs Maximaux Monotones. North-Holland, Amsterdam (1973).

7. Colli, P., Frémond, M., \& Visintin, A. Thermo-mechanical evolution of shape memory alloys. Quarterly Appl. Math. 48, (1990) 31-47.

8. Colli, P. \& Sprekels, J. Global existence for a three-dimensional model for the thermo-mechanical evolution of shape memory alloys. Nonlinear Anal. 18, (1992) 873-888.

9. Colli, P. \& Visintin, A. On a class of doubly nonlinear evolution equations. Comm. P.D.E. 15, (1990) $737-756$.

10. DUBINSKIĬ, YU. A. Weak convergence in nonlinear elliptic and parabolic equations. (In Russian). Mat. Sbornik 67 (109), (1965) 609-642.

11. Fattorini, H. O. Infinite Dimensional Optimization and Control Theory. Cambridge Univ. Press, Cambridge (1999).

12. Frémond, M. \& MiYazaki, S. Shape Memory Alloys. Springer, Vienna (1996).

13. HiLl, R. A variational principle of maximum plastic work in classical plasticity. Q. J. Mech. Appl. Math. 1, (1948) 18-28.

14. Hoffmann, K.-H., NiezgódKa, M., \& Zheng, Songmu Existence and uniqueness of global solutions to an extended model of the dynamical development in shape memory alloys. Nonlinear Analysis, Th. Meth. Appl. 15, (1990) 977-990.

15. Hoffmann, K.-H. \& RoubíčEK, T. Thermomechanical evolution of a microstructure. Quarterly Appl. Math. 52, (1994) 721-737.

16. HuO, Y. \& MÜLleR, I. Nonequilibrium thermodynamics of pseudoelasticity. Continuum Mech. Thermodyn. 5, (1993) 163-204.

17. JAMES, R. D. Hysteresis in phase transformations, Preprint No. 1361. IMA, Minneapolis (1995).

18. KREJČÍ, P. Evolution variational inequalities and multidimentional hysteresis operators, Preprint No. 432. WIAS, Berlin (1998).

19. LapczyK, I., Rajagopal, K. R., \& SRinivasa, A. R. Deformation twinning during impactnumerical calculations using a constitutive theory based on multiple natural configuration. Computational Mechanics 21, (1998) 20-27.

20. Mielke, A. \& Theil, F. A mathematical model for rate-independent phase transformations with hysteresis. In: Alder, H.-D., BALEAn, R. \& FARWIG, R. (eds), Models of continuum mechanics in analysis and engineering. pp. 117-129. Shaker, Aachen (1999).

21. Mielke, A. \& Theil, F. On rate-independent hysteresis models. Nonlinear Diff. Eq. Appl. (2001), to appear.

22. Mielke, A., Theil, F., \& LevitAs, V. I. Mathematical formulation of quasistatic phase transformations with friction using an extremum principle, Preprint No. A8. Uni., Hannover (1998).

23. MÜLlER, S. Variational models for microstructure and phase transitions, Lect. notes no. 2. Max-Planck Institute, Leipzig (1998).

24. NiezGódKA, M. \& SpReKels, J. Existence of solutions for a mathematical model of structural phase transitions in shape memory alloys. Math. Methods in Appl. Sci. 10, (1988) 197-223.

25. Powell, M. J. D. ZQPCVX, A Fortran subroutine for convex programming, Report DAMTP/1983/NA17, Univ. of Cambridge (1983).

26. Rajagopal, K. R. \& SRinivasa, A. R. On the inelastic behavior of solids: 1. Twinning. Int. J. Plasticity 11, (1995) 653-678. 
27. RAJAgOpal, K. R. \& SRInivasa, A. R. Inelastic behavior of materials: II. Energies associated with twinning. Int. J. Plasticity 13, (1997) 1-35.

28. RAJAGOPAL, K. R. \& SRInivaSA, A. R. On the thermomechanics of shape memory wires. Zeitschrift angew. Math. Phys. 50, (1999) 459-496.

29. Ren, X. \& TRUSKinOvsky, L. Finite scale microstructures in nonlocal elasticity. J. Elasticity 59, (2000) 319-355.

30. Rogers, R. \& Truskinovsky, L. Discretization and hysteresis. Physica B 233, (1997) 370-375.

31. RoubíčEK, T. Evolution of a microstructure: a convexified model. Math. Methods in the Applied Sciences 16, (1993) 625-642.

32. RoubíčEK, T. Finite element approximation of a microstructure evolution. Math. Methods in the Applied Sciences 17, (1994) 377-393.

33. RoubíčEK, T. Relaxation in Optimization Theory and Variational Calculus. W. de Gruyter, Berlin (1997).

34. RoubíČEK, T. Dissipative evolution of microstructure in shape memory alloys. In: BUnGARTZ, H.-J., Hoppe, R. H. W. \& Zenger, C. (eds), Lectures on Applied Mathematics. pp. 45-63. Springer, Berlin (2000).

35. RoubíčEK, T. \& KRUŽíK, M. Numerical treatment of microstructure evolution modelling. In: Bock, H. G. et al., (eds), ENUMATH 97. pp. 532-539. World Scientific, Singapore (1998).

36. RoubíčEK, T. \& ŠVERÁK, V. Nonexistence of solutions in nonconvex multidimensional variational problems. J. Convex Anal. 7, (2000) 427-436.

37. RYBKA, P. Dynamical modelling of phase transitions by means of viscoelasticity in many dimensions. Proc. Royal Soc. Edinburgh 121A, (1992) 101-138.

38. ŠILHAVÝ, M.: On the pseudoelastic hysteresis. (a preprint)

39. Simon, J. Compact sets in the space $L^{p}(0, T ; B)$. Annali di Mat. Pura Applic. 146, (1987) 65-96.

40. SRINIVASA, A. R. personal communication, Texas A\& M University, (January 1999).

41. Visintin, A. Models of Phase Transitions. Birkhäuser, Boston (1996).

42. Yosida, K. \& HewitT, E. Finitely additive measures. Trans. Amer. Math. Soc. 72, (1952) $46-66$. 